Review Article

\title{
A Review on the Low-Dimensional and Hybridized Nanostructured Diamond Films
}

\author{
Hongdong Li, Shaoheng Cheng, Jia Li, and Jie Song \\ State Key Lab of Superhard Materials, Jilin University, Changchun 130012, China \\ Correspondence should be addressed to Hongdong Li; hdli@jlu.edu.cn
}

Received 30 April 2015; Accepted 1 July 2015

Academic Editor: Jose Alvarez

Copyright (C) 2015 Hongdong Li et al. This is an open access article distributed under the Creative Commons Attribution License, which permits unrestricted use, distribution, and reproduction in any medium, provided the original work is properly cited.

In the last decade, besides the breakthrough of high-rate growth of chemical vapor deposited single-crystal diamonds, numerous nanostructured diamond films have been rapidly developed in the research fields of the diamond-based sciences and industrial applications. The low-dimensional diamonds of two-dimensional atomic-thick nanofilms and nanostructural diamond on the surface of bulk diamond films have been theoretically and experimentally investigated. In addition, the diamond-related hybrid nanostructures of n-type oxide/p-type diamond and n-type nitride/p-type diamond, having high performance physical and chemical properties, are proposed for further applications. In this review, we first briefly introduce the three categories of diamond nanostructures and then outline the current advances in these topics, including their design, fabrication, characterization, and properties. Finally, we address the remaining challenges in the research field and the future activities.

\section{Introduction}

Diamond has been deeply investigated and widely applied in numerous fields because of its unique properties of wide band gap, high thermal conductivity, hardness, good chemical stability, chemical inertness, high temperature stability, inherent biocompatibility, and so forth, which make it suitable for use in various high performance optoelectronic devices. In addition to conventional bulk diamond crystals (e.g., chemical vapor deposited (CVD) single crystal diamonds and polycrystalline diamond films), the fabrication and properties of diamond-related nanostructures have attracted a significant amount of interest [1]. Designing new nanostructures of diamond and exploiting novel properties become an important hot topic in current researches. With increasing the interest of two-dimensional (2D) graphene in the recent decade since 2004 [2], the atomic-thickness 2D diamond nanofilms without and with surface functional terminations have emerged [3-7]. These nanofilms have smaller band gaps and varying magnetic properties with respect to those of the bulk diamond, which are strongly dependent on layer number $(n)$ and surface functionalizations. This new category of diamond has been constructed, though the most researches are based on theoretical calculations thus far. The other low-dimensional nanodiamonds (e.g., nanowires, nanopits, and nanotips) have been experimentally generated by "topdown" approach $[8,9]$ on the surface of bulk single-crystal diamonds and/or polycrystalline diamond films and show some excellent optical, electronic, and biological properties. The third category of heterojunction structures consists of various nanosized semiconducting materials and bulk polycrystalline diamond films (single-crystal diamonds), which are favorable for achieving high performance optoelectronic devices. The nanosized materials are generally of n-type metallic compounds (such as zinc oxide $\mathrm{ZnO}[10,11]$, titania $\mathrm{TiO}_{2}$ [12], and gallium nitride $\mathrm{GaN}$ [13]) deposited on the p-type diamond. In this review, we discuss and summarize the recent developments of fabrications, characterizations, properties, and potential applications based on the above three diamond-related topics. These works in the recent 10 years will be beneficial to the future development of diamond material with more novel structures and better properties for applications.

\section{Two-Dimensional Diamond Nanofilms}

In recent decades, besides the conventional bulk carbonaceous materials (i.e., graphite, diamond, and disordered 


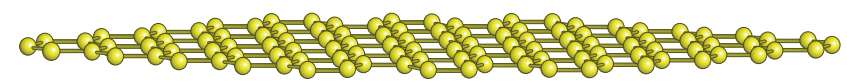

(a)
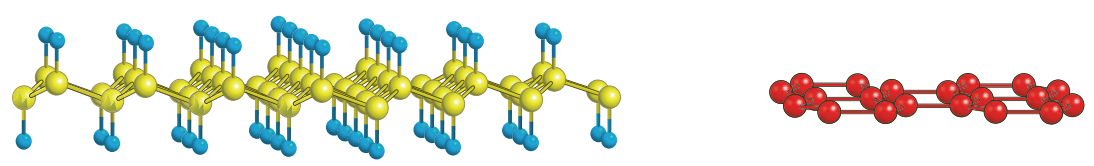

(b)
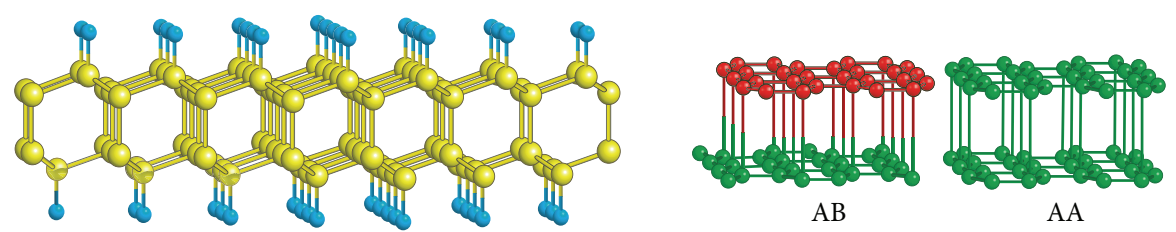

(c)

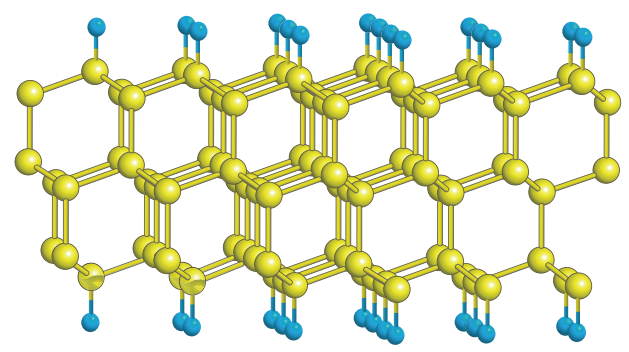

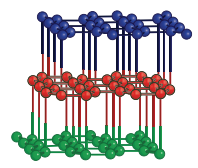

$\mathrm{ABC}$

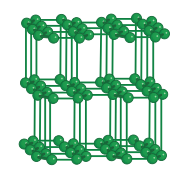

AAA

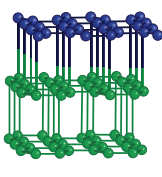

AAC

(d)

Figure 1: Atomic geometry of the studied two-dimensional (2D) carbon nanofilms: (a) graphene; (b) graphane; (c) diamane of two layers; and (d) diamane of three layers. Carbon (hydrogen) atoms are marked with yellow (blue). For each diamane, possible stacking sequences of carbon layers are presented on the right side [19].

carbon), various new nanostructures (such as fullerene [14], carbon nanotubes [15], graphene [2], and diamond clusters [16]) have appeared in the family of carbon. In 2007, full hydrogenation on both sides of graphene (with $\mathrm{C}_{x} \mathrm{H}_{x}$ ), referred to as "graphane," was theoretically predicted [5], and this configuration with buckling feature is similar to that of hydrogenated diamond (111) planes. Chernozatonskii et al. considered a $\mathrm{C}_{2} \mathrm{H}$ nanostructure [6] based on bilayer graphene to constitute a $2 \mathrm{D}$ hydrogenated $\langle 111\rangle$ diamond film with a thickness of less than $1 \mathrm{~nm}$, which is called "diamane." It was theatrically demonstrated that $\mathrm{C}_{2} \mathrm{H}$ diamane is more stable than $\mathrm{CH}$ graphane. Both hydrogenated diamondlike films (graphane and diamane) have relatively wide band gaps narrower than that of bulk diamond within $1 \mathrm{eV}[5,17$, 18]. These early works open up a new research area of low dimensional diamond. Figure 1 shows the atomic geometry of the 2D carbon nanofilms [19]. The authors summarized the calculated formation energy and band gap width of different hydrocarbon structures as a function of hydrogen content.

Zhu et al. investigated the layer number $(n)$ dependent formation and electronic properties of hydrogenated $2 \mathrm{D}$ diamond nanofilms originated from few-layer graphene [3]. The band gap monotonically decreases with the increasing thickness (i.e., $n$ ), reflecting a quantum confinement effect (Figure 2(a)). Due to the presence of hydrogen passivated surface, the tunable gaps are localized in a region lower than that of bulk diamond. Based on building blocks of $\mathrm{C}_{35}$ and $\mathrm{C}_{84}$ with octahedral morphology, Li and Zhao [7] described the structurally optimized few-layer (six-layer) 2D diamondrelated nanostructure (nanoflake) having a diamond matrix covered with graphite fragments. As shown in Figure 3, the two outmost layers were delaminated and partially graphitized having a nonsymmetrical reconstruction. It revealed that the stability of these 2D diamond nanofilms generally requires termination with functional groups or reconstructive $\mathrm{sp}^{2}$-carbon surface.

Different from the previous first-principles calculations based on few-layer graphene and building blocks of carbon clusters, Li et al. [4] performed $n$-dependent structural optimization of 2D diamond nanofilms (Figure 4) beginning from the original diamond (111) layers without termination of foreign atoms, cleaved from bulk cubic diamond with single-dangling-bond surface. At $n \leq 5$, the films are relaxed into a few graphene layers, whereas for $6 \leq n \leq$ 11 , a gradient-graphite-diamond-like (GGDL) structure with gradient changes of interplanar spacing and buckling feature is predicted. A threshold $n=12$ is determined to realize the thinnest two-dimensional (111)-oriented diamond film. For $n \geq 12$, the diamond phase is energetically stabilized. The effects of semihydrogenation $(\mathrm{SH})$ and full hydrogenation $(\mathrm{FH})$ on the structural evolution and properties of $2 \mathrm{D}$ diamond nanofilms were further investigated [20]. Both of the hydrogenation processes play an important role in stabilizing the $2 \mathrm{D}$ diamond structures. Interestingly, for the $\mathrm{SH}$ cases, 


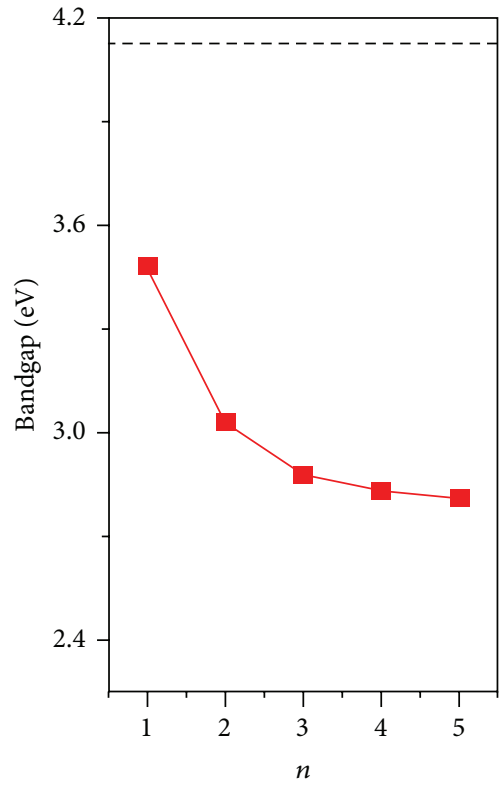

(a)

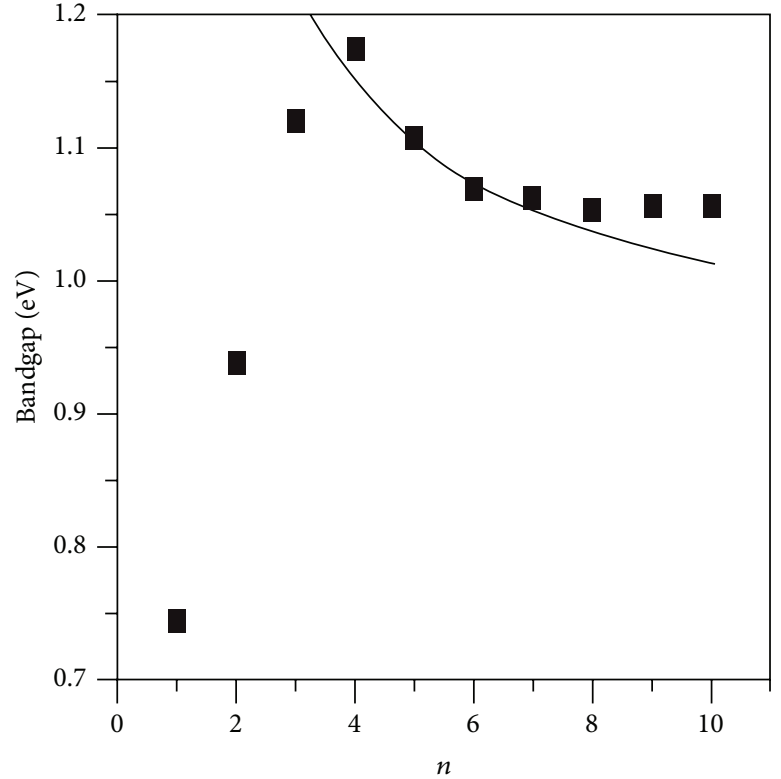

(b)

Figure 2: (a) Band gaps of fully hydrogenated two-dimensional (2D) cubic diamond nanofilms as a function of layer number ( $n$ ). The dashed line corresponds to the band gap of bulk diamond [3]. (b) Band gaps of semihydrogenated 2D diamond nanofilms as a function of $n$. The solid line represents the fitted curve [20].

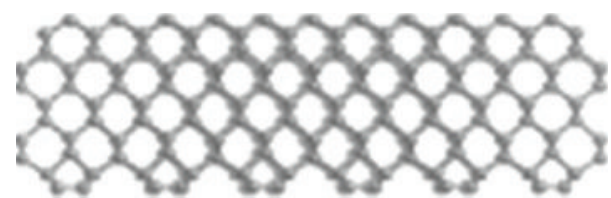

(a)

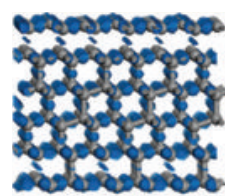

(b)

Figure 3: The initial structure (a) and the deformation electron density picture (b) for the two-dimensional (2D) diamond nanoflakes with 6 layers after structural geometry optimization generated from cuboctahedral nanodiamond $\mathrm{C}_{142}$ as the building block [7].

the spin-related band gaps are in an infrared region of 0.74$1.17 \mathrm{eV}$ (Figure 2(b)), and a ferrimagnetism characteristic is presented determined by the unpaired electrons on the outmost 3 layers on nonhydrogenated side (Figure 5) which are strongly dependent on $n$. Layer-by-layer partial density of states (PDOS) revealed that the carbons with dangling bonds near the surface determine the tuning electronic and magnetic properties of 2D diamond nanofilms $[4,20]$.

For the novel ultrathin diamond nanofilms, it is desirable to know how defects and dopants can tune the corresponding electronic properties. Fyta [21] recently theoretically investigated the electronic structure of diamane by introducing dopants of boron, nitrogen, sulfur atoms, and defects of nitrogen vacancy (NV) with different charged states. As a general remark (summarized in Table 1), adding dopants or defects closes the energy gap of ideal diamane films as these provide available electronic states within that gap. The amount of band gap reduction is associated with the chosen dopants or the charge state of the NV defect. Adding a dopant with a large size mismatches to the lattice and leads to a tendency to stabilize the doping atoms at surface, and, accordingly, the electrons are localized around the dopant
TABle 1: Bond lengths (in $\AA$ ), the HOMO-LUMO gap $\left(E_{g}\right.$, in eV), and the relative difference (in \%) of this gap with respect to that of the ideal diamane film $\left(E_{g}^{0}\right)$ for different diamane films [21].

\begin{tabular}{lllllllc}
\hline Structure & $r_{b}^{(1,2)}$ & $r_{b}^{(1,3)}$ & $r_{b}^{(1,4)}$ & $r_{b}^{(1,5)}$ & $r_{b}^{(\mathrm{CH})}$ & $E_{g}$ & $\Delta E_{g} / E_{g}^{0}$ \\
\hline diam & 1.550 & 1.534 & 1.532 & 1.533 & 1.126 & 4.05 & \\
N-diam & 1.554 & 1.523 & 1.523 & 1.523 & 1.136 & 3.99 & 1.5 \\
B-diam & 1.613 & 1.620 & 1.620 & 1.563 & 1.130 & 3.71 & 8.4 \\
S-diam & 2.901 & 1.793 & 1.792 & 1.792 & 1.130 & 1.40 & 65.4 \\
$\mathrm{NV}^{0}$-diam & & 1.452 & 1.457 & 1.452 & 1.144 & 0.67 & 83.4 \\
$\mathrm{NV}^{-}$-diam & & 1.447 & 1.447 & 1.447 & 1.151 & 0.61 & 84.9 \\
$\mathrm{NV}^{+}$-diam & & 1.458 & 1.457 & 1.459 & 1.140 & 0.95 & 76.5 \\
vac-diam & & 1.536 & 1.624 & 1.536 & 1.117 & 1.40 & 65.4 \\
\hline
\end{tabular}

site. Furthermore, the spins of these NV centers in diamane close to the surface could prove much more efficiency than $\mathrm{NV}$ center in bulk diamond in nanomagnetic resonance imaging (nano-MRI) applications. The results are relevant to potential applications of diamane films in nanoelectronics.

Besides the calculations, finding a possible fabrication route of scaled 2D diamond nanofilms is more necessary for 


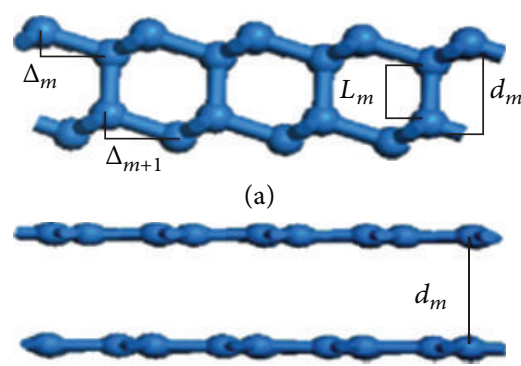

$\left(a^{\prime}\right)$

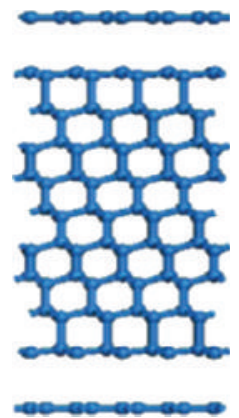

(c)

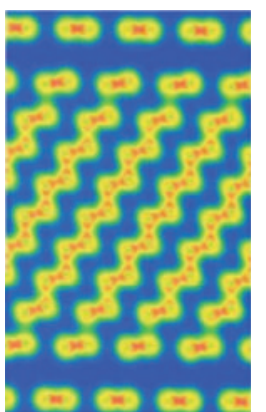

$\left(c^{\prime}\right)$

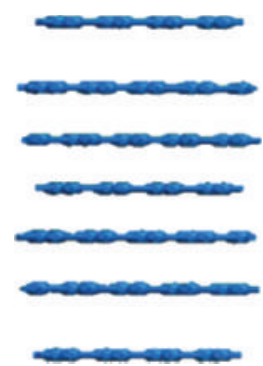

(b)

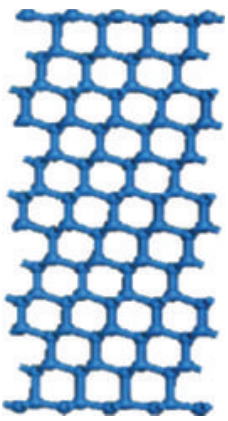

(d)

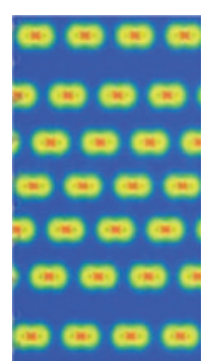

$\left(b^{\prime}\right)$

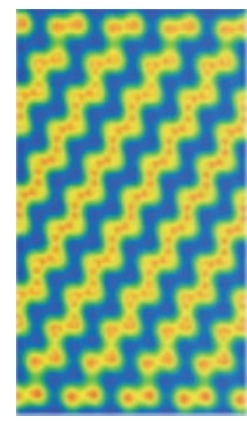

$\left(\mathrm{d}^{\prime}\right)$

Figure 4: Structural evolution from original two-dimensional (2D) atomically few layer diamonds. The side views of the optimized configurations and the corresponding electronic charge density features for the cases of $n=2\left(\mathrm{a}, \mathrm{a}^{\prime}\right), n=7\left(\mathrm{~b}, \mathrm{~b}^{\prime}\right), n=11\left(\mathrm{c}, \mathrm{c}^{\prime}\right)$, and $n=12\left(\mathrm{~d}, \mathrm{~d}^{\prime}\right)$, respectively. The thicknesses are normalized [4].

the practical application. Li et al. $[4,20]$ pointed out possible routes to experimentally realize the freestanding layered 2D diamond by laser ablation from bulk diamond and/or find these nanosized diamond layers from ball milling and detonation products. CVD technique is a suitable method to obtain mass products of the atomically thin diamond films. To obtain the hydrogenated diamond films, treating in $\mathrm{H}_{2}$ ambient (at high temperature and/or in $\mathrm{H}_{2}$ plasma) for $2 \mathrm{D}$ pure nanofilms or transition from hydrogenated few-layer graphene under high pressure might be feasible. Through the first-principles density functional theory calculations, Odkhuu et al. [22] investigated the transformation of a few layers of graphene (with hydrogenation or fluorination on the outer surface of the top graphene layer) into $\mathrm{sp}^{3}$-bonded thin overlayers (also named as diamond-like layer) on metallic surfaces (Co, Ni, and $\mathrm{Cu}$ ) (Figure 6). Strong hybridization between the $\mathrm{sp}^{3}$ dangling bonds orbitals and the metallic surface $\mathrm{d}_{z}^{2}$ orbitals stabilizes the $\mathrm{sp}^{3}$-bonded carbon layers. And, it was speculated that the metal/ $/ \mathrm{sp}^{3}$ carbon interfaces might be a new candidate for superconductivity study. The computational results suggest a route to experimental realization of large-area ultrathin $\mathrm{sp}^{3}$-bonded carbon films on metal surfaces.

Most recently, Kvashnin et al. [23] further theoretically demonstrated that few-layer graphene can undergo phase transformation into thin diamond film under reduced or no pressure, if the process is facilitated by hydrogenation of the surfaces. Such a "chemically induced phase transition" is inherently nanoscale phenomenon, when the surface conditions directly affect thermodynamics, and the transition pressure depends greatly on film thickness. The role of finite diameter of graphene flakes and possible formation of the diamond films with the (110) surface was described. Later, Antipina and Sorokin [24] used $\mathrm{H}, \mathrm{H}_{2}, \mathrm{~F}, \mathrm{~F}_{2}, \mathrm{H}_{2} \mathrm{O}$, and $\mathrm{NH}_{3}$ as the adsorbates to produce high-quality films of diamond from few-layer graphene at different temperatures and pressures by ab initio calculations. The functionalized cubic or hexagonal diamond films with specific surface would show well-defined properties.

Summarily, the above results underline the strong differences of the structures and properties of those atomically thick 2D diamond nanofilms compared to bulk diamond, and these should have implications in various potential nanoelectronical applications.

\section{Nanostructural Surface of Bulk Diamond Film}

As diamond is an important material for its outstanding properties in many aspects, fabrication of micro/nanodiamond (films) is essential to enhance the intrinsic properties and to find new properties for fully realizing the potentials of diamond in numerous research fields. The designs of formatting diamond micrometer- and nano-structures generally include two approaches of "bottom-up" and "top-down". In this review, we focus on the "top-down" method that has widely been applied because of its controllability, variety, and large area. Up to now, various top-down nanofabrication techniques have been explored for well-designed diamond nanostructures [8, 9, 25-37]. 

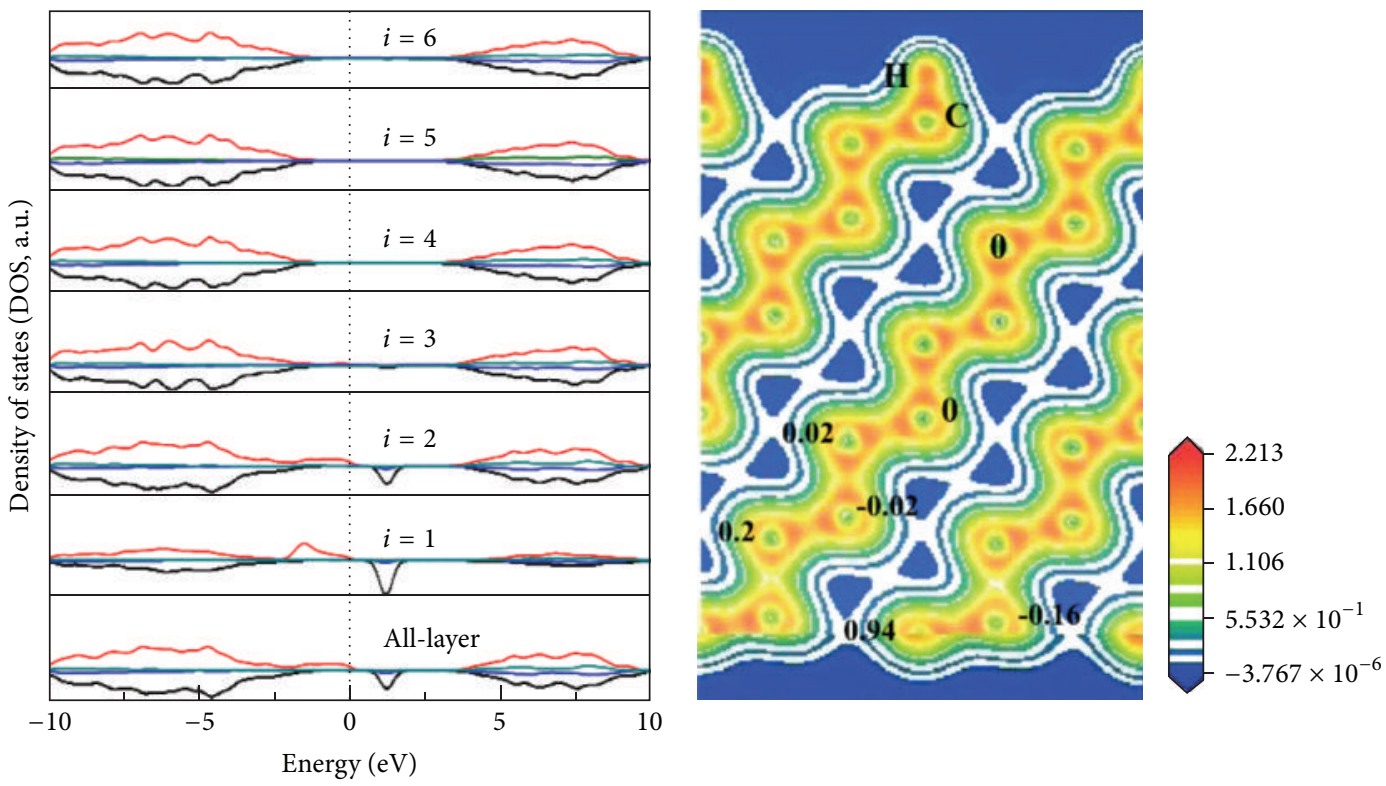

FIgURE 5: Partial density of states (PDOS) and spin density picture of the calculated semihydrogenated two-dimensional (2D) diamond nanofilm with $n=6$. Layer-by-layer PDOS ( $i=1$ to 6 ) are taken from the outmost layer on one side to the opposite side in turn. The red (green) and black (blue) lines are corresponding PDOS of $p(s)$-orbital electrons with spin-up and spin-down, respectively. The Fermi level is set to $0 \mathrm{eV}$. The intensities of the total and partial DOS are normalized. The pictures of spin density are labeled with the corresponding magnetic moments around the atoms [20].

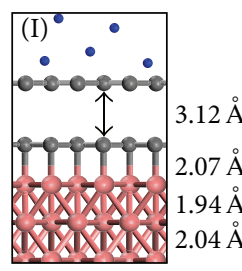

(a)

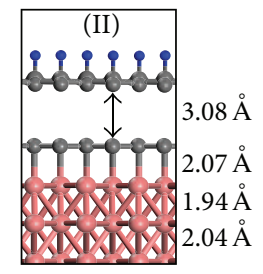

(b)

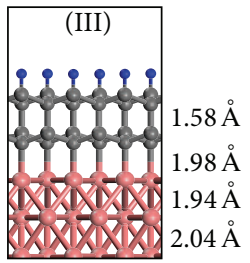

(c)

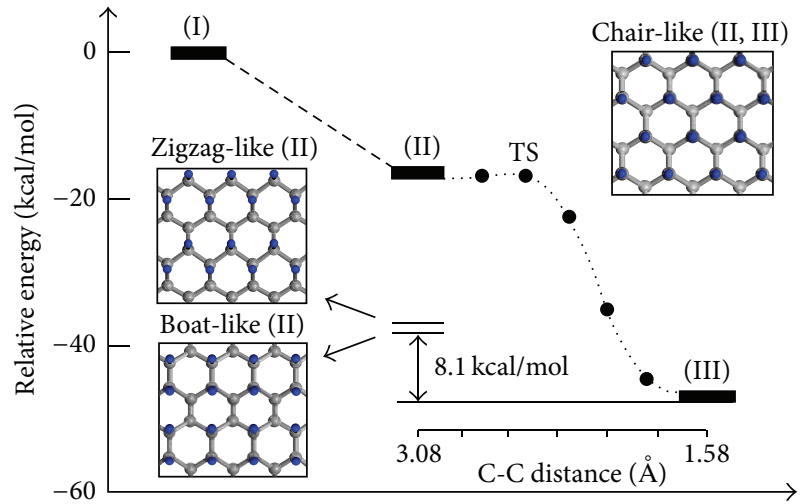

(d)

Figure 6: The energetics of conversion of bilayer graphene into $\mathrm{sp}^{3}$ carbon film on metal surface. For details see [22].

In 1997, Shiomi [8] realized nanosized columnar structure from polycrystalline diamond films by reactive ion etching (RIE) in $\mathrm{O}_{2} / \mathrm{CF}_{4}$ plasma and the examined electron field emission characteristics were improved, which triggered activities of fabricating nanostructures on diamond films. Masuda et al. (in 2000 [9]) introduced ordered through-hole anodic porous alumina membranes employed on top of both undoped and boron-doped p-type CVD diamond films and then fabricated nanohoneycomb diamond film using a radiofrequency driven (at $13.56 \mathrm{MHz}$ ) oxygen plasma etching treatment (Figure 7). Fine structures with a high degree of ordering can be obtained over large areas $\left(\sim 1 \mathrm{~cm}^{2}\right)$ controlled by changing the geometrical structure of the porous alumina membrane mask. The through holes in the masks have high aspect ratios (depth-to-diameter) compared to those used in conventional lithography. Using the nanohoneycomb diamond as electrodes, it is found that the capacitance values increased depending on the pore dimensions, and a film of pore diameter $400 \mathrm{~nm}$ and pore depth $3 \mu \mathrm{m}$ exhibited a 400 -fold increase in the capacitance $\left(3.91 \times 10^{-3} \mathrm{~F} \mathrm{~cm}^{-2}\right)$ in comparison to an as-deposited surface film (Figure 7(c)) [25].

By depositing gold nanodots as etching masks on the as-grown polycrystalline nanodiamond (ND) and microdiamond (MD) films, Zou et al. (in 2008 [26]) fabricated highdensity uniform diamond nanopillar arrays by employing bias-assisted RIE in a hydrogen/argon plasma (Figure 8). The formation of nanopillar structure is associated with the directional physical etching/sputtering by ion bombardment 


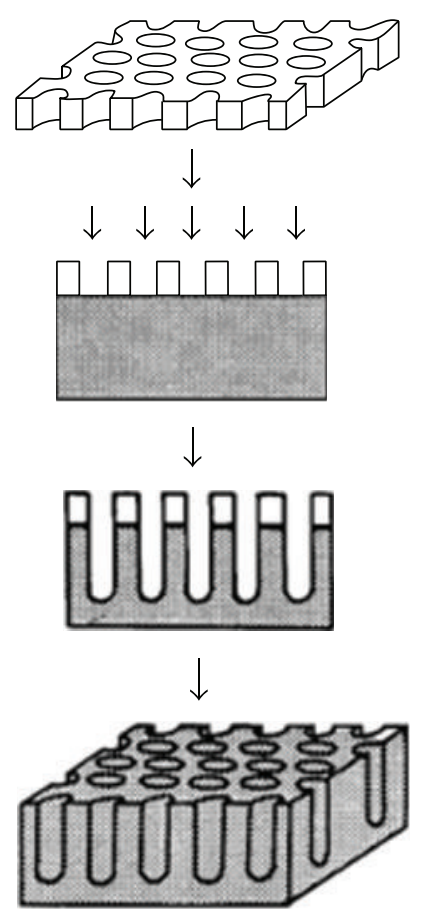

(a)

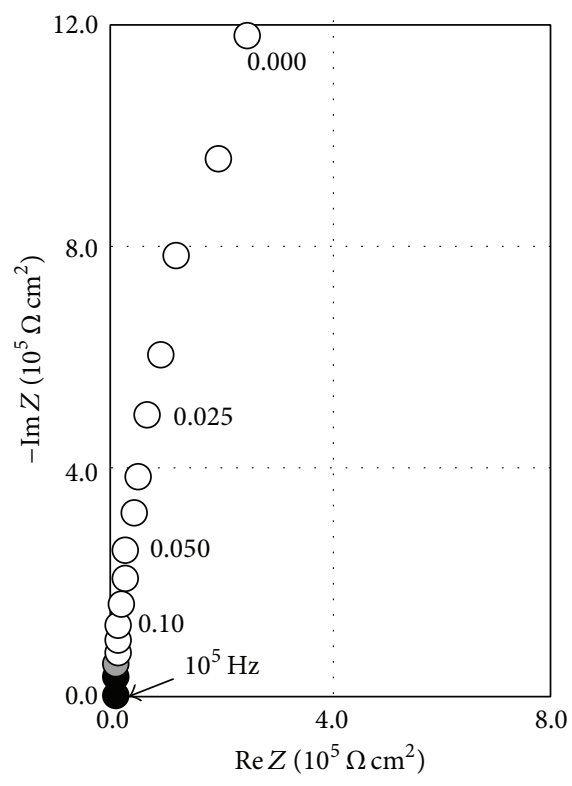

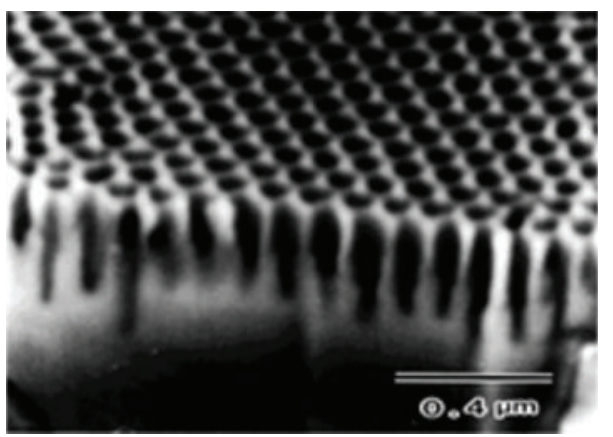

(b)

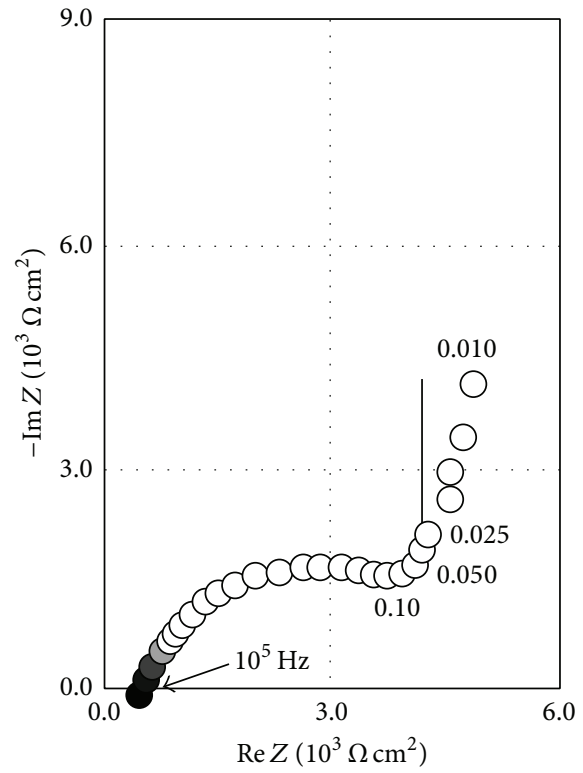

(c)

Figure 7: (a) Schematic diagram of the fabrication procedure for the diamond nanohoneycomb. (b) Cross-sectional view of the diamond nanohoneycomb. (c) Complex-plane plots of the impedance for electrodes of as-deposited diamond (left) and pore types $400 \mathrm{~nm} \times 3 \mu \mathrm{m}$ (right), at $10.4 \mathrm{~V}$ versus $\mathrm{Ag} / \mathrm{AgCl}[9,25]$.

and selective chemical etching of $\mathrm{sp}^{2}$ carbons by reactive hydrogen atoms and ions. The density and geometry of these nanopillars were determined by the initial structure of diamond films and reactive ion etching conditions. These nanopillars have potential applications in high performance diamond-based biomedical and chemical sensors and in mechanical and thermal management.
Using the similar nanoassembled Au nanoparticles (NPs) as the mask, another type of hybrid structure of $\mathrm{Au}$ $\mathrm{NP} /$ diamond-nanopit has been fabricated on single-crystal diamond by oxygen plasma etching (Figure 9(a)) [27-29], where the diamond-nanopits are different to diamond nanopillars and/or nanowires arrays generated from polycrystalline diamond films having abundant grain boundaries 


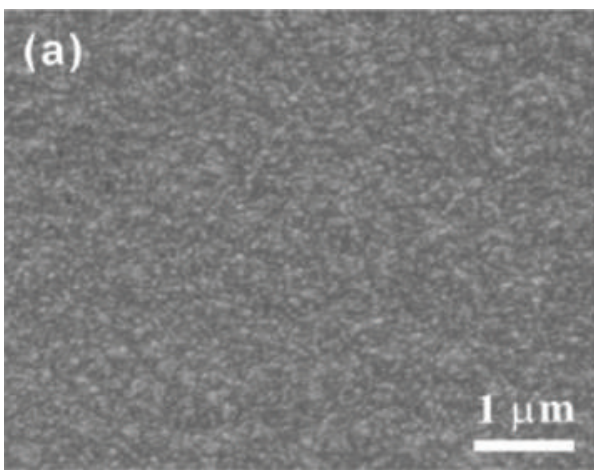

(a)

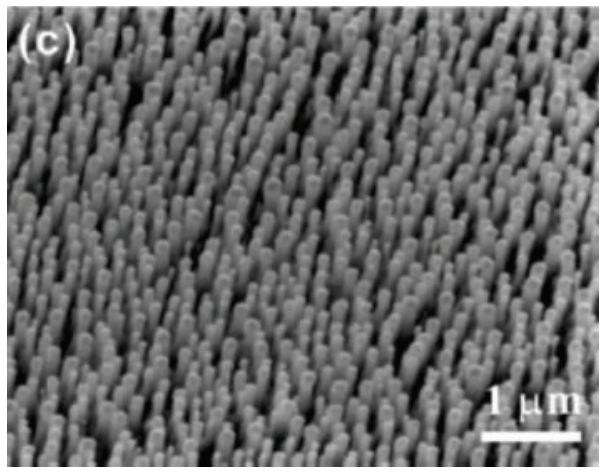

(c)

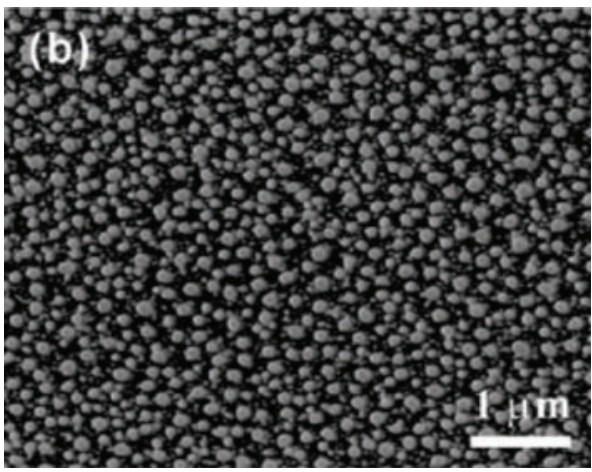

(b)

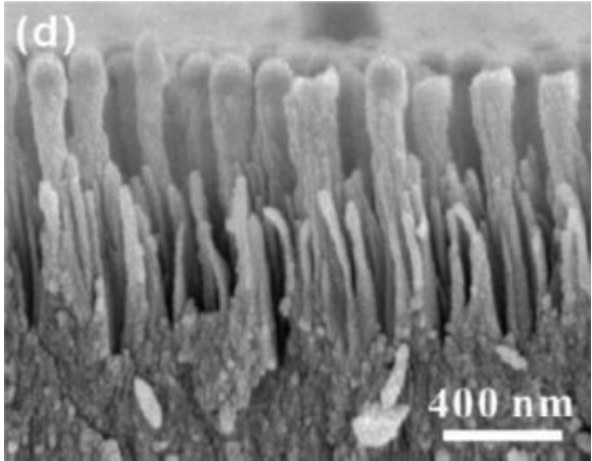

(d)

FIGURE 8: SEM morphologies showing (a) the nanocrystalline diamond film with a flat surface, (b) the gold nanodots formed on the nanocrystalline diamond surface, and (c) nanocrystalline diamond film with nanopillar array collected by sample tilting and (d) from the cross section [26].
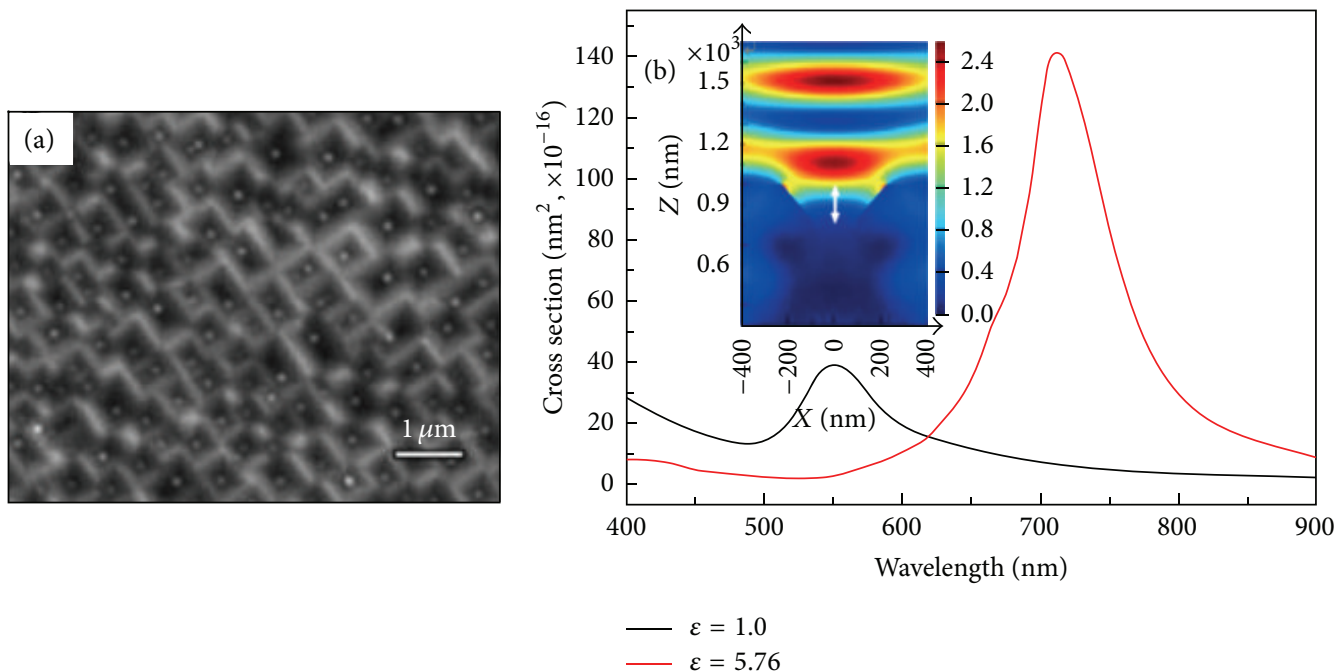

FIGURE 9: (a) The planar SEM image of the surface of single crystal diamond after oxygen plasma etching for $60 \mathrm{~s}$ having nanopits infilled with Au nanoparticles. (b) Calculated LSPR scattering cross section spectra of a semiellipsoidal Au NP placed in air (dark) and on diamond (red), showing the resonance peaks at 550 (in air) and $718 \mathrm{~nm}$ (on diamond). The inset of (b) reveals the cross-sectional electric field intensity $\left(|E|^{2}\right)$ distribution in a diamond-nanopit $[27,28]$. 
(1) Diamond nanoparticle seeding

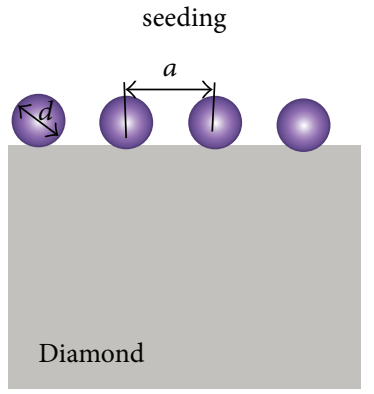

(2) $\operatorname{RIE}(t=0)$

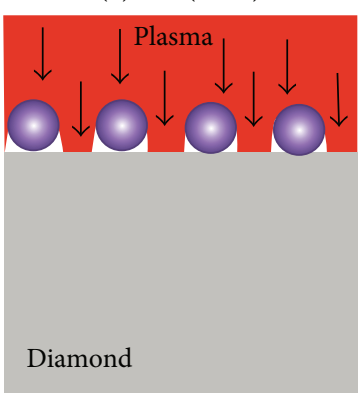

(3) RIE $(t>0)$

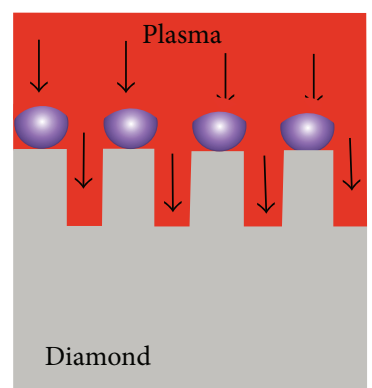

(4) Final surface structure

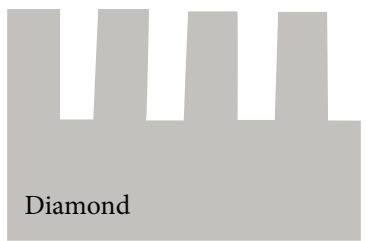

(a)

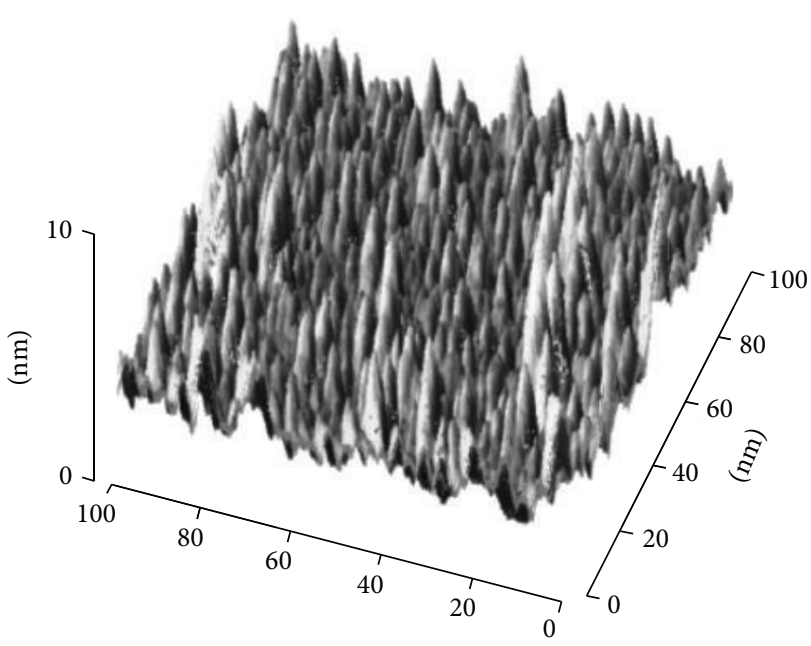

(b)

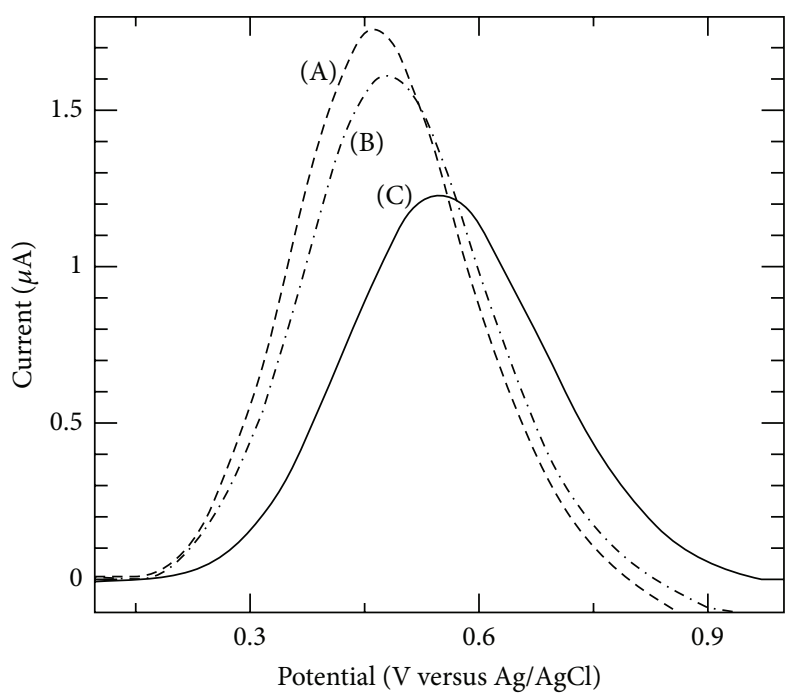

(c)

FIGURE 10: (a) Schematic plots of fabrication of diamond nanowires by a reactive ion etching (RIE). (b) Typical AFM image of the fabricated diamond nanowires. (c) Differential pulse voltammograms of $1.0 \mathrm{mM} \mathrm{Fe}(\mathrm{CN})_{6}{ }^{3-/ 4-}$ in $\mathrm{pH} 7.4$ phosphate buffer as detected on diamond nanowires after marker ss-DNA immobilization (curve (A)), after exposure to single-based mismatched DNA (10 nM; curve (B)), and after exposure to complementary target DNA $(10 \mathrm{nM}$; curve $(\mathrm{C}))[30,31]$.

[26]. The three-dimensional finite-difference time-domain (FDTD) simulations revealed strong localized surface plasmon resonance (LSPR) scattering and enhanced electric field exhibited by this hybrid nanoassembled-metal/diamondnanopit (Figure 9(b)). Experimentally, with excitations at 633 and $830 \mathrm{~nm}$ close to the calculated LSPR wavelength, the photoluminescence (PL) intensities of the peak at $738 \mathrm{~nm}$ originating from the single-photon source of silicon vacancy $(\mathrm{SiV})$ centers in diamond were significantly enhanced by factors of $\sim 100$ and $\sim 50$, respectively, with respect to that from normal Si-doped diamond without the hybrid structure [27]. This structure could also realize a larger surface enhanced Raman scattering (SERS) enhancement factor [28]. Because of the unique advantages of diamond, the diamond-nanopit structure can serve as a robust structural template for fabricating various membranes with pyramid-structured surface [29].

The other strategy of mask is using diamond nanoparticles [30-32]. By depositing diamond nanoparticles and following a RIE in an $\mathrm{O}_{2} / \mathrm{CF}_{4}$ gas mixture, Yang et al. [30] realized diamond nanowires on boron-doped single-crystalline
CVD diamond films after a top-down procedure (Figure 10). As an electrode, the active area of diamond nanowires has been significantly enhanced. Furthermore, the tip-modified diamond nanowires are promising with respect to biosensor applications. When used as DNA sensor, the sensitivity is about 100 to 1000 times better, compared to published data available in the literature, where comparable DNA strands have been used with comparable redox mediator molecules on planar gold electrodes, gold nanowires, and diamond [31]. Based on the similar method, Lu et al. fabricated vertically aligned diamond nanoneedles on polycrystalline diamond nanofilms [32]. Gas sensing measurements with the diamond nanoneedles were performed at room temperature for both reducing $\left(\mathrm{NH}_{3}\right)$ and oxidizing $\left(\mathrm{NO}_{2}\right)$ gases. Due to the increased surface area-to-volume ratio and nanotip effect of the diamond nanoneedles, great enhancements of chemical sensing ability and sensitivity were presented compared with the unmodified nanodiamond films.

The development of a robust light source that emits one photon at a time will allow new technologies such as secure communication through quantum cryptography. 


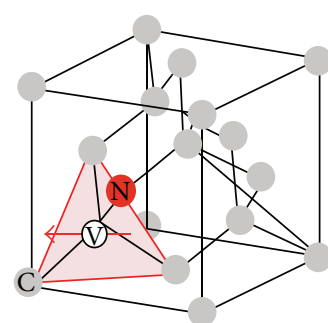

(a)

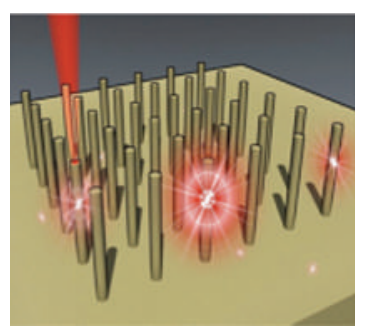

(b)

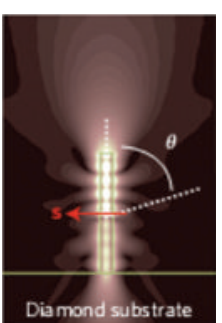

(d)

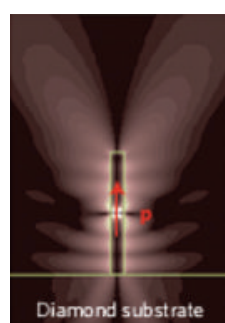

(e)

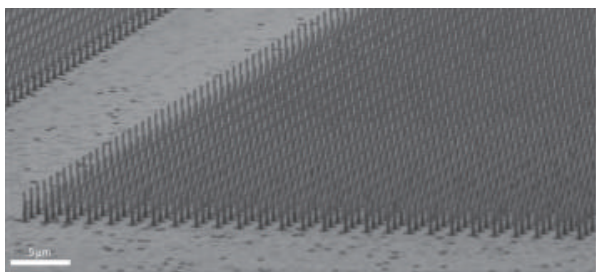

(c)

FIGURE 11: Single-photon source based on an NV centre in a diamond nanowire. For details see [34].

Luminescent centers in diamond (e.g., nitrogen vacancy (NV) centers) have recently emerged as a stable source offering spin quantum bits with optical readout. Hausmann et al. [33] and Babinec et al. [34] demonstrated a single-photon source composed of a NV center in a diamond nanowire on the (100) crystal plane (Figure 11), which produces ten times greater flux than bulk diamond devices, while using ten times less power. The diamond nanowires were fabricated from a mask patterned diamond crystal by RIE with oxygen plasma. This result, as well as that for $\mathrm{SiV}$ enhancement [27], enables a new class of devices for photonic and quantum information processing based on nanostructured diamond and could have a broader impact in nanoelectromechanical systems (NEMS), sensing, and scanning probe microscopy.

For further investigating the advanced single-photon properties of NV center, Jiang et al. [35] developed an overlay patterning method for fast, designable, and controllable fabrication of various three-dimensional (3D) diamond micro/nanostructures on (100) plane with focused-ion-beam (FIB) milling. Diamond solid immersion lens and nanopillar with NV center placed at the designed location could enable efficient single-photon collection by overcoming the total internal reflection at the diamond/air interface. The enhancements of about tenfold of the single photons collection rate, well-reserved long electron spin dephasing time, and single-photon emission properties have been obtained. In 2014, Neu et al. [36] fabricated nanophotonic structures of diamond nanopillars on (111)-oriented single-crystalline CVD diamond. The authors demonstrated that this crystal orientation offers optimal coupling of NV center emission to the nanopillar mode and might thus be advantageous over previous approaches with (100) crystal orientation. High saturated fluorescence count rates around $10^{6}$ counts per second were realized based on these nanopillars.

Combining procedures of carbon ion implantation, photolithography, and electron beam lithography and RIE, Liao et al. [37] designed and fabricated the suspended single-crystal diamond nanowires with well-controlled dimensions in batches for NEMS (Figure 12). All-single-crystal diamond NEM switches were demonstrated using these nanowires with high reproducibility, high controllability, repeated switching, and no stiction. Diamond switches not only overcome the stiction and abrasion problems in existing electromechanical switches, but also circumvent the shallow doping problem. The concept and technology in this work would be helpful for promoting the rapid developments of the next generation of high performance NEMS.

\section{Diamond-Related Nanoheterojunction}

Combining oxide or nitride semiconductors with diamond substrates attracts great attention mainly motivated by the lack of efficient p-type doping in the oxides (nitrides) and n-type doping in diamond, respectively. The complementary configuration of these wide band gap materials with p-type boron-doped diamond and n-type oxides $\left(\mathrm{ZnO}, \mathrm{TiO}_{2}\right)$ and n-type nitrides ( $\mathrm{GaN}, \mathrm{AlN}$ ) has been most widely studied recently $[10-13,38-53]$. Generally, growth of oxides and nitrides films on diamond remains challenging due to the high difference in the thermal expansion coefficients and large lattice mismatch (leading to structural imperfections) and relatively less efficient performance of devices. Compared with continuous bulk films, the low-dimensional structures (e.g., nanorods, nanowires, and nanotubes) with a high ratio of surface to volume, defect-free, and enhanced quantum effect are expected to yield much better structural quality and do not suffer from thermal mismatch on diamond, consequently improving the performances of the hybridized structural devices. In this part, we focus on three structures of $\mathrm{ZnO} /$ diamond, $\mathrm{TiO}_{2}$ /diamond, and $\mathrm{GaN} /$ diamond, where the oxides and nitrides are of nanosized feature.

$\mathrm{ZnO}$ is an important semiconductor having unique properties of wide direct wide band gap $(3.37 \mathrm{eV})$ and large 


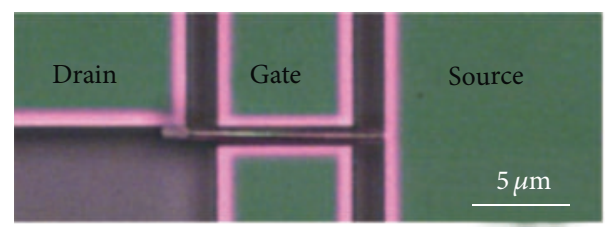

(a)

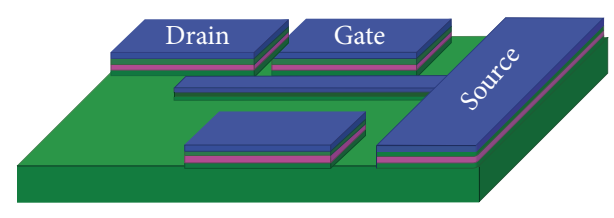

(b)

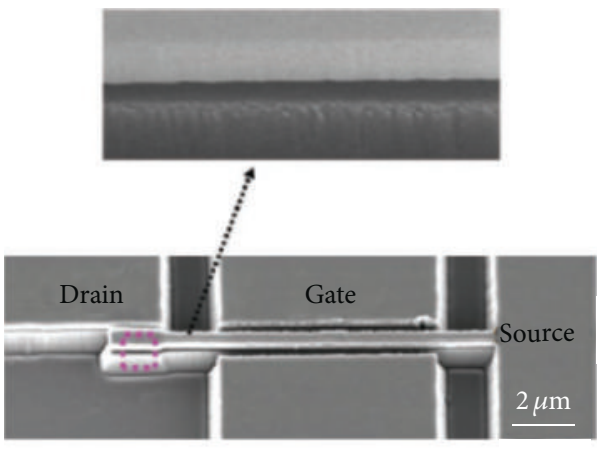

(c)

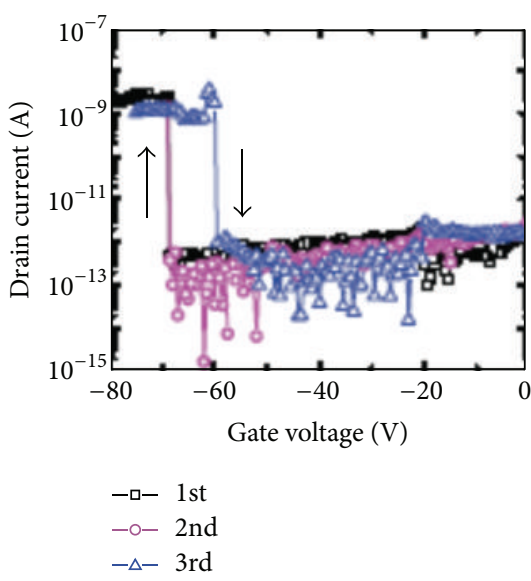

(d)

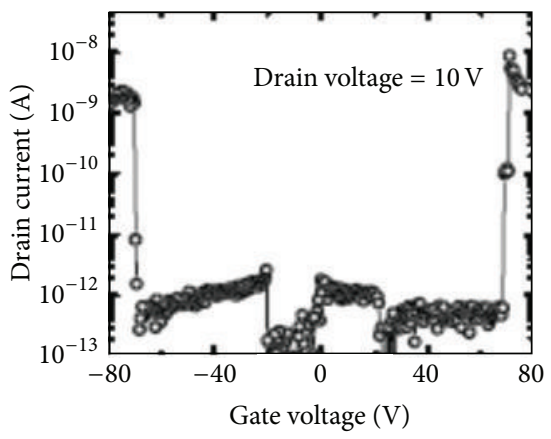

(e)

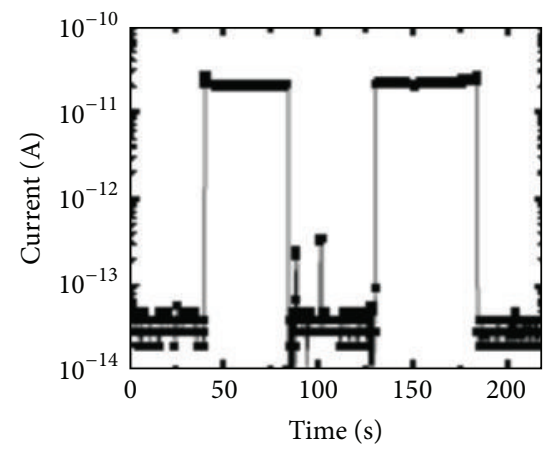

(f)

FIGURE 12: (a) Optical image of all-single-crystal diamond 3T-NEM switch in ON state. The dimensions of the cantilever are $14 \mu \mathrm{m}$ in length and $400 \mathrm{~nm}$ in width. The gate-source gap is $400 \mathrm{~nm}$. (b) Schematic diagram of the switch. (c) SEM image of the switch viewed at a titled angle of $20^{\circ}$. (d) Repeated and hysteresis switching behavior of the device controlled by the gate voltage. (e) Gate voltage-drain current dependence of a similar switch. (f) Time dependence of the switch at a gate voltage of $75 \mathrm{~V}$ and a drain voltage of $5 \mathrm{~V}$ [37].

exciton binding energy $(60 \mathrm{meV})$ at room temperature. The combined structures of diamond and $\mathrm{ZnO}$ have been paid more attention to fabricate semiconductor heterojunctions [52]. Previously, the existing form of $\mathrm{ZnO}$ was generally performed as film deposited on diamond wafer [53]. In recent decade, $\mathrm{ZnO}$-related nanostructures and the corresponding nanooptoelectronic devices have been extensively studied [54]. The heterojunctions based on $\mathrm{ZnO}$ nanostructures were generally grown on silicon [55], GaN [56], sapphire [57], and diamond [11, 39, 41]. Experimentally, the performances of the diamond-related biosensor electrodes have been significantly improved by modifying $\mathrm{ZnO}$ nanorods (NRs) (Figure 13) synthesized using the sol-gel method [10]. Li's group reported the growth of $\mathrm{ZnO}$ NRs on CVD diamond by thermal vapor transport [11, 38-40] and hydrothermal methods [41]. Using the former method, in the initial growth status, the semispherical $\mathrm{ZnO}$ nuclei were preferably deposited near the growth steps on the terraces and the boundaries of diamond grains. With increasing the growth time, the [0001] orientated $\mathrm{ZnO}$ nanorods with large aspect ratio appeared and further covered the whole diamond film, as shown in Figure 14. Furthermore, the epitaxial relation between the (0001) $\mathrm{ZnO}$ and (111) diamond is proposed following
(0001)[11̄̄0] $\mathrm{ZnO} / /(111)[1 \overline{1} 0]$ diamond or $(0001)[10 \overline{1} 0]$ $\mathrm{ZnO} / /(111)[1 \overline{1} 0]$ diamond $[39,42]$, while the relation between the (0001) $\mathrm{ZnO}$ and (100) diamond is mainly of (0001)[0001] ZnO//(101)[101] diamond [39].

As doped and/or unintentionally doped $\mathrm{ZnO}$ is generally $\mathrm{n}$-type semiconductor and p-type diamond is easy to be realized by boron doping, the hybrid $\mathrm{n}-\mathrm{ZnO} \mathrm{NRs} / \mathrm{p}$-diamond heterojunctions have been constructed [11,39]. For a n$\mathrm{ZnO} \mathrm{NRs} / \mathrm{p}$-diamond heterojunction with degenerated $\mathrm{p}$ type diamond (heavily B-doped diamond having a higher carrier concentration at $10^{20} / \mathrm{cm}^{3}$ order of magnitude) especially, a negative differential resistance (NDR) phenomenon was evidently presented in the corresponding $I-V$ plot (Figure 15), which can be attributed to the tunneling current in the structure [39]. Since both diamond and $\mathrm{ZnO}$ are important high-temperature semiconductor materials, the temperature-dependent electrical properties of the n$\mathrm{ZnO} \mathrm{NRs/p-diamond} \mathrm{heterojunctions} \mathrm{were} \mathrm{studied} \mathrm{and} \mathrm{the}$ corresponding parameters varied at different temperatures [11]. At relatively high temperature, the ohmic behavior and the carrier injection has been improved. And at higher voltages, the injected current of the heterojunction follows the trap-free space-charge-limited current (SCLC) law with 


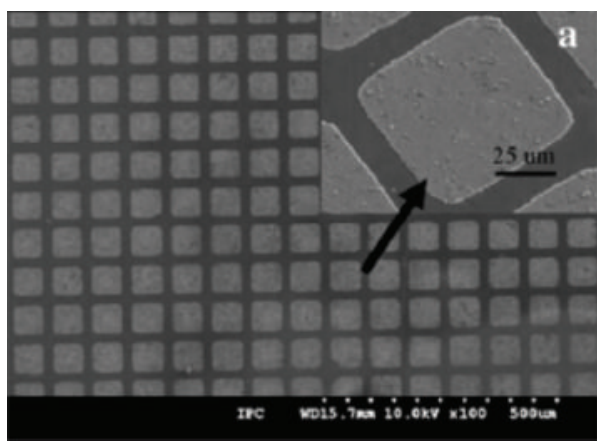

(a)

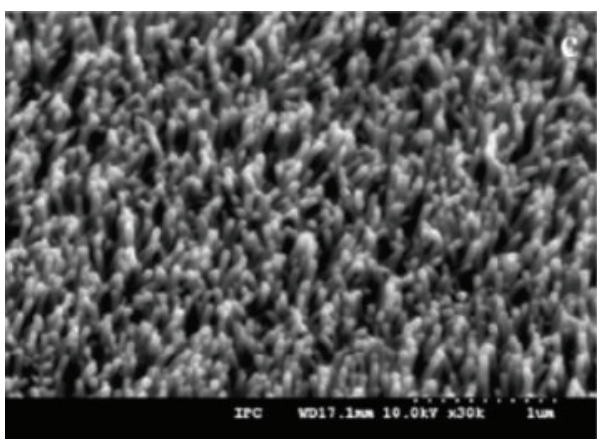

(c)

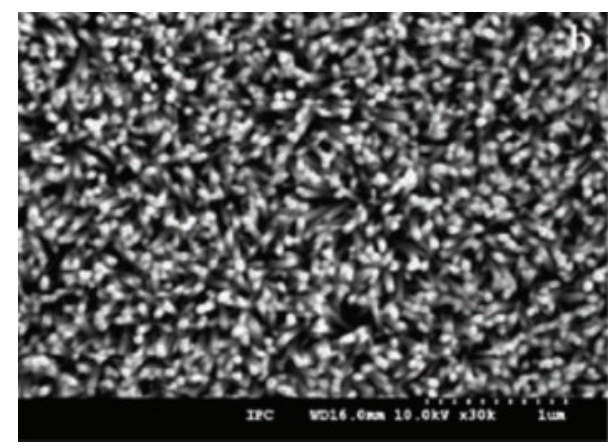

(b)

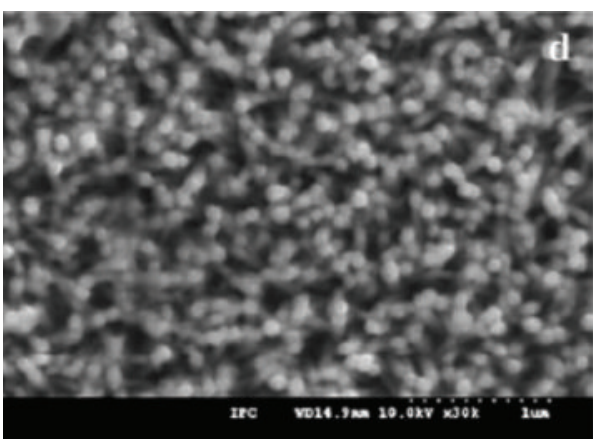

(d)

FIGURE 13: SEM images of (a) the ZnO nanorod microarrays on boron-doped nanodiamond thin film surfaces (the inset shows the enlarged image of a square-shaped $\mathrm{ZnO}$ nanorod array), (b) top view of $\mathrm{ZnO}$ nanorod arrays, and (c) tilt view of $\mathrm{ZnO}$ nanorod arrays and (d) functional $\mathrm{ZnO}$ nanorod arrays [10].

the exponent close to 2 because the traps states will be filled by larger thermally activated carriers. These results emphasize the suitability for fabricating $\mathrm{n}-\mathrm{ZnO} / \mathrm{p}$-diamond-based high frequency, high power, and high temperature optoelectronic nanodevices.

Another important metal oxide of $\mathrm{TiO}_{2}$ has attracted considerable attention because it is especially suitable for applications in the fields of photocatalytic degradation of pollutants, hydrogen generation from photoelectrocatalytic water splitting, dye-sensitized solar cells, and so forth [58]. The applications of $\mathrm{TiO}_{2}$ as photocatalysts are encumbered to a certain extent by the relatively low quantum yield that is normally caused due to rapid recombination of photogenerated electrons and holes. In order to enhance the quantum yield, a heterojunction structure has been further introduced because the heterojunction could provide a potential driving force (the internal electrostatic potential in the space charge region) to reduce the recombination of photogenerated charge carriers. Highly efficient photocatalysts have been realized by n-type anatase $\mathrm{TiO}_{2}$ film joined to CVD p-type boron-doped diamond (BDD) [43, 44], which has unique properties of wide electrochemical potential window, long-term response stability, high level of mechanical strength, and chemical corrosion resistance. Compared with continuous $\mathrm{TiO}_{2}$ films, low-dimensional $\mathrm{TiO}_{2}$ structures (e.g., nanotubes, nanorods), which possess a high ratio of surface to volume and provide a convenient way for photogenerated carriers to transfer to the reaction surfaces, lead to improved photocatalytic efficiency [45,
46]. In [47, 48], $\mathrm{TiO}_{2}$ nanoparticles (nanotubes) have been deposited on BDD film by a spin coating method for applications in electrochemical reactivity (electro- and bioelectrocatalysis). By a liquid phase deposition (LPD) method using a $\mathrm{ZnO}$ nanorod template, the vertical anatase $\mathrm{TiO}_{2}$ nanotube (TiNT) arrays were directly grown on a CVD BDD film, and the fabricated n-type TiNT/p-type BDD (n-TiNT/p-BDD) heterojunctions showed highly efficient photocatalytic activities for the decomposition of azo dye C.I. reactive yellow 15 (RY15) [12]. The increased ability to separate photogenerated charge carriers by the pn junction (n-TiNT/p-BDD) would enhance the photocatalytic activity. Additionally, the TiNT array structure provides more active positions for the photocatalytic processes exposed to UV light, and the loose TiNTs are favorable for permitting more UV light to reach the BDD side through TiNTs with respect to the case of $\mathrm{TiO}_{2}$ film on BDD.

In 2015, different to depositing $\mathrm{TiO}_{2}$ on diamond, Siuzdak et al. [49] reported on novel composite nanostructure of thin BDD films (thickness of $\sim 200-500 \mathrm{~nm}$ ) grown on top of asfabricated $\mathrm{TiO}_{2}$ nanotubes (Figure 16). The nanostructures made of $\mathrm{BDD}$-modified $\mathrm{TiO}_{2}$ nanotubes showed an increase in activity and performance when used as electrodes in electrochemical environments. The substantial improvement of electrochemical performance and the excellent rate capability could be attributed to the synergistic effect of $\mathrm{TiO}_{2}$ treatment in $\mathrm{CH}_{4} / \mathrm{H}_{2}$ plasma and the high electrical conductivity of BDD layers. 


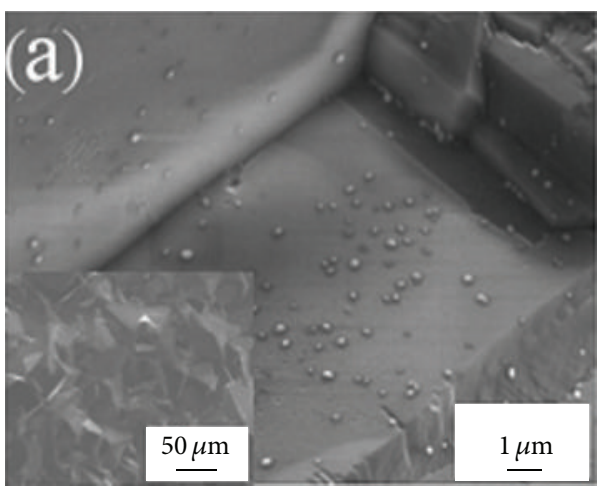

(a)

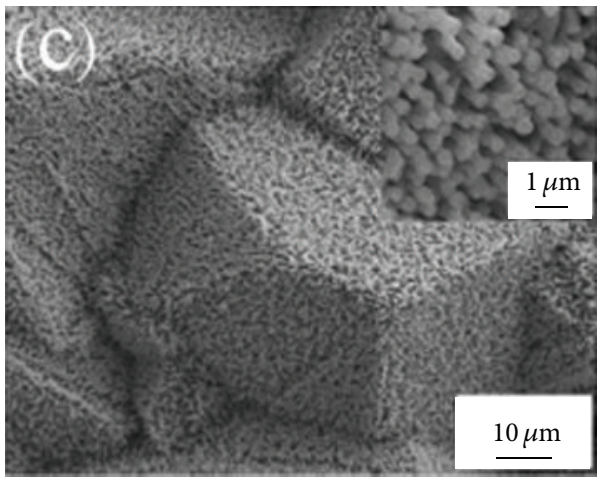

(c)

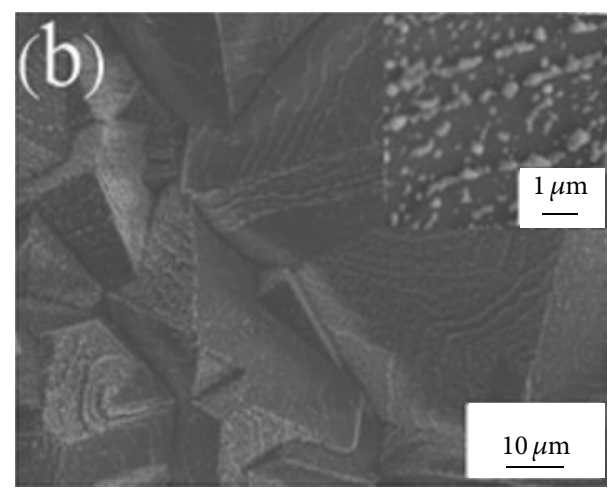

(b)

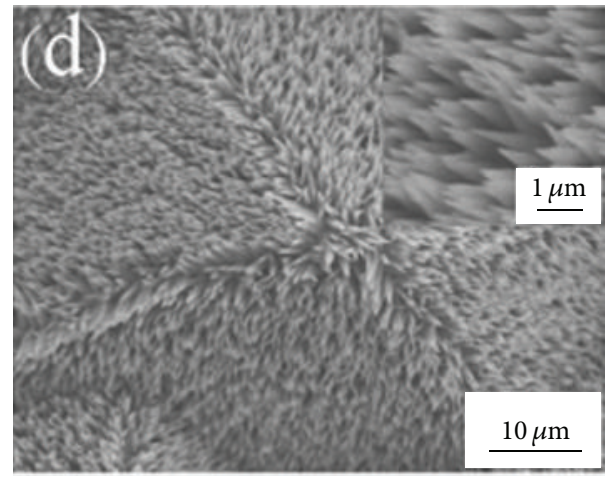

(d)

FiguRE 14: SEM images of $\mathrm{ZnO}$ nanostructures synthesized on microcrystalline diamond films for $0.5 \mathrm{~min}(\mathrm{a}), 2 \mathrm{~min}$ (b), and 5 min ((c) and (d)). The inset of (a) is the image of bird's-eye view. The insets of (b)-(d) are the high-magnitude images of ZnO NRs [40].

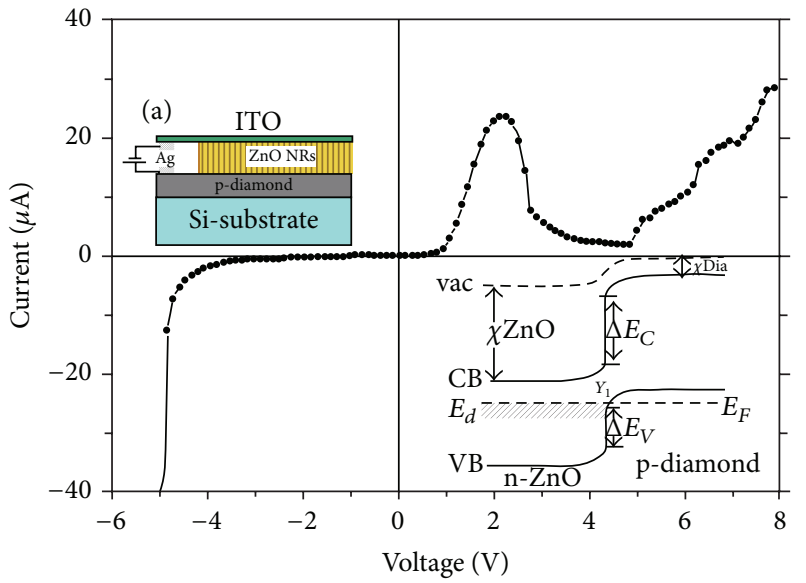

Figure 15: $I-V$ characteristic of the $\mathrm{n}-\mathrm{ZnO} \mathrm{NR} / \mathrm{p}$-diamond heterojunction constructed with degenerated p-diamond, showing a negative differential resistance phenomenon. The insets are the schematic diagram of the pn heterojunction (upper left) and the energy band diagram of the heterojunction at thermal equilibrium (lower right) [39].

In recent years, the field of GaN-based nanowires (NWs) has made substantial progress as a possible alternative to planar structures for light-emitting diodes, laser diodes, sensing applications, photovoltaics, and catalysis. Schuster et al. $[13,50,51]$ reported on the fabrication, characterization, and properties of the low-dimensional GaN structures on diamond, which provide important information for realizing high performance optoelectronic nanodevices in the UVspectral range and the electrical control of NV centers in diamond. By plasma-assisted molecular beam epitaxy, the self-assembled epitaxial GaN NWs with an excellent crystalline quality were vertically grown catalyst-free on (111) single crystal diamond (Figure 17). A strong and sharp excitonic emission in the PL spectrum reveals excellent optical properties superior to state-of-the-art GaN NWs on silicon substrates [13]. The position-controlled growth of GaN NWs on diamond substrates with the help of structured titanium masks was demonstrated, and a transition from a nanowire toward a nanotube growth mode was observed [50]. The controlled fabrication of $\mathrm{GaN}$ nanotube arrays will be of interest for applications benefiting from a high surfaceto-volume ratio. Furthermore, the authors addressed the different aspects of GaN nanowire doping ( $\mathrm{Si}$ and $\mathrm{Mg}$ ) [51]. The GaN NWs density and strains state, distributions of dopant concentration and charge carriers, and optical properties related to the doping in NWs have been investigated. The results help to understand and control the mechanisms of doping in GaN nanostructures on diamond to intentionally tailor their electric and optical properties for device fabrication. 


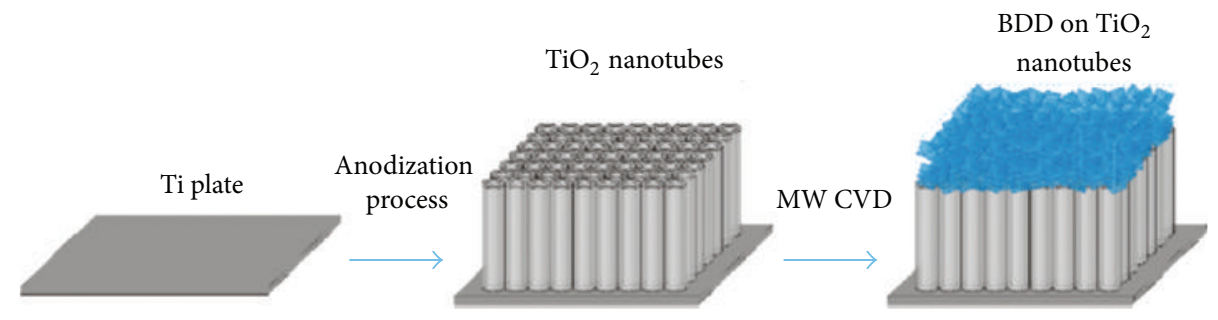

FIGURE 16: Formation scheme of composite $\mathrm{TiO}_{2}$ nanotube/boron-doped diamond (BDD) electrodes [49].
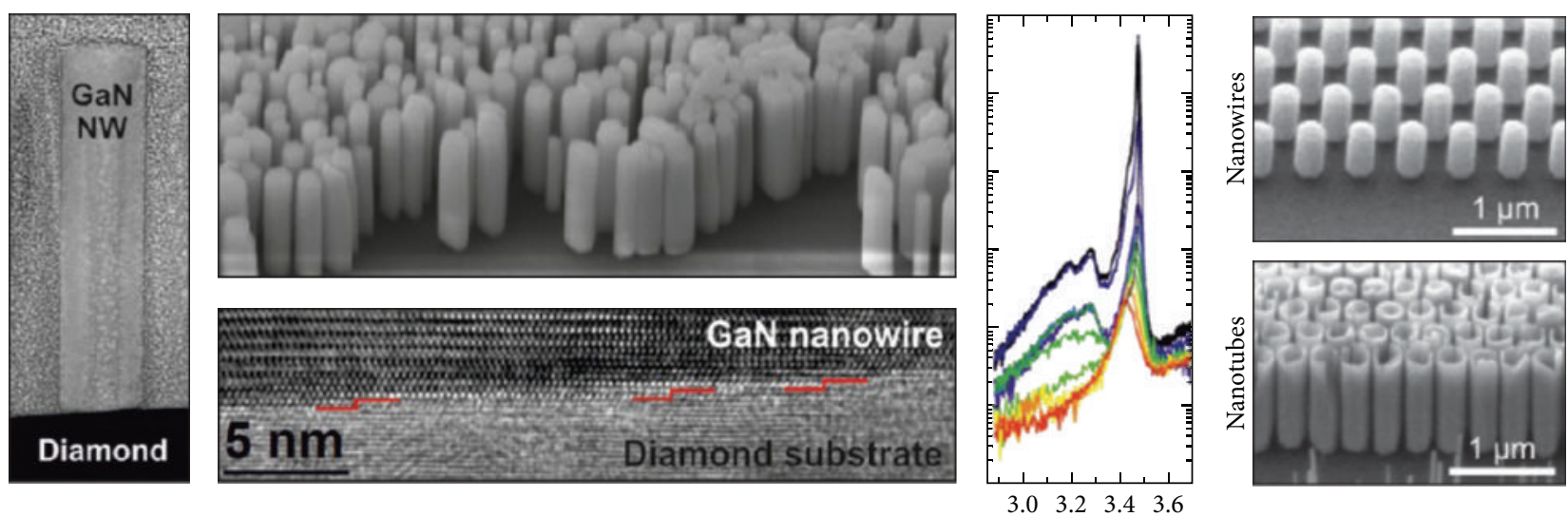

FIGURE 17: From left to right: cross-section high-angle annular dark-field STEM of an exemplary GaN NW on diamond substrate; tilted-view SEM images of as-grown GaN NWs on diamond (top), HRTEM image of the interface region (bottom); temperature-dependent PL spectra of GaN NWs on diamond with typical NBE shift due to band gap shrinkage [13]; selective area grown GaN NW arrays without (top) and with a hole (bottom) [50].

\section{Conclusion and Outlook}

In this review, we gave a very recent overview of diamondrelated nanostructures, especially including the three types developed in the current decade. The atomically thick $2 \mathrm{D}$ fewlayer diamond nanofilms with and without varying surface functionalization have been theoretically predicted, and the corresponding optimized structures and the electronic and magnetic properties are calculated showing differences from that of bulk diamond. The challenge of those 2D nanofilms is to find a feasible route to experimentally realize in large sizes (e.g., more than tens and hundreds of micrometers) for practical application. Some possible approaches have been mentioned in the text. As for the nanostructural surface of diamond films, which are generally obtained by top-down approach following various etching processes (with designed mask patterns), great achievements have been made in plasmonic enhancement, single-photon source, electron field emission, and biologic sensing for the potential applications. The technologies of feasibility, low cost, large scale, high accurate controllability, and novel structures are quite desirable to be developed to meet the growing demand. The heterostructures combined of nanosized ntype oxides (nitrides) and bulk p-type diamond have been experimentally fabricated to overcome the lattice mismatch, lack of efficient p-type doping in the oxides and nitrides and n-type doping in diamond, and structural imperfections in the interface region. However, depositing high quality and highly orientated nanosized oxides (nitrides) on (100)-oriented diamond, which is of high quality grown by CVD process, is difficult to be realized due to the large lattice mismatch between the hexagonal/tetragonal oxides (nitrides) and the cubic (100) diamond facet. Suitable buffer layer and/or optimized growth conditions (e.g., temperature, bias enhanced nucleation, and/or pretreated substrate) should be provided to achieve the goal. In short, this review reveals the current key issues, opportunities, and challenges facing present and future researches on diamond material with various nanostructures for advancing in commercial applications.

\section{Conflict of Interests}

The authors declare that there is no conflict of interests regarding the publication of this paper.

\section{Acknowledgment}

This work was supported by the National Natural Science Foundation of China (NSFC) (Grant no. 51472105).

\section{References}

[1] D. M. Gruen, O. A. Shenderova, and A. Y. Vul, Synthesis, Properties and Applications of Ultrananocrystalline Diamond, vol. 192 of NATO Science Series, Springer Netherlands, 2005. 
[2] K. S. Novoselov, A. K. Geim, S. V. Morozov et al., "Electric field effect in atomically thin carbon films," Science, vol. 306, no. 5696, pp. 666-669, 2004.

[3] L. Y. Zhu, H. Hu, Q. Chen, S. D. Wang, J. L. Wang, and F. Ding, "Formation and electronic properties of hydrogenated few layer graphene," Nanotechnology, vol. 22, no. 18, Article ID 185202, 2011.

[4] H. D. Li, J. Li, Z. G. Wang, and G. T. Zou, "Layer numberdependent structural evolution of two-dimensional diamond films," Chemical Physics Letters, vol. 550, pp. 130-133, 2012.

[5] J. O. Sofo, A. S. Chaudhari, and G. D. Barber, "Graphane: a two-dimensional hydrocarbon," Physical Review B-Condensed Matter and Materials Physics, vol. 75, no. 15, Article ID 153401, 2007.

[6] L. A. Chernozatonskii, P. B. Sorokin, A. G. Kvashnin, and D. G. Kvashnin, "Diamond-like $\mathrm{C}_{2} \mathrm{H}$ nanolayer, diamane: simulation of the structure and properties," JETP Letters, vol. 90, no. 2, pp. 134-138, 2009.

[7] L. S. Li and X. Zhao, "Transformation between different hybridized bonding structures in two-dimensional diamondbased materials," The Journal of Physical Chemistry C, vol. 115, no. 45, pp. 22168-22179, 2011.

[8] H. Shiomi, "Reactive ion etching of diamond in $\mathrm{O}_{2}$ and $\mathrm{CF}_{4}$ plasma, and fabrication of porous diamond for field emitter cathodes," Japanese Journal of Applied Physics, vol. 36, no. 12, pp. 7745-7748, 1997.

[9] H. Masuda, M. Watanabe, K. Yasni, D. Tryk, T. Rao, and A. Fujishima, "Fabrication of a nanoslructured diamond honeycomb film," Advanced Materials, vol. 12, no. 6, pp. 444-447, 2000.

[10] J. W. Zhao, D. H. Wu, and J. F. Zhi, "A novel tyrosinase biosensor based on biofunctional $\mathrm{ZnO}$ nanorod microarrays on the nanocrystalline diamond electrode for detection of phenolic compounds," Bioelectrochemistry, vol. 75, no. 1, pp. 44-49, 2009.

[11] D. D. Sang, H. D. Li, S. H. Cheng, Q. L. Wang, Q. Yu, and Y. Z. Yang, "Electrical transport behavior of $\mathrm{n}-\mathrm{ZnO}$ nanorods/pdiamond heterojunction device at higher temperatures," Journal of Applied Physics, vol. 112, no. 3, Article ID 036101, 2012.

[12] J. J. Yuan, H. D. Li, S. Y. Gao, Y. H. Lin, and H. Y. Li, "A facile route to n-type $\mathrm{TiO}_{2}$-nanotube/p-type boron-doped-diamond heterojunction for highly efficient photocatalysts," Chemical Communications, vol. 46, no. 18, pp. 3119-3121, 2010.

[13] F. Schuster, F. Furtmayr, R. Zamani et al., "Self-assembled GaN nanowires on diamond," Nano Letters, vol. 12, no. 5, pp. 21992204, 2012.

[14] H. W. Kroto, J. R. Heath, S. C. O'Brien, R. F. Curl, and R. E. Smalley, " $\mathrm{C}_{60}$ : buckminsterfullerene," Nature, vol. 318 , no. 6042, pp. 162-163, 1985.

[15] S. Iijima, "Helical microtubules of graphitic carbon," Nature, vol. 354, no. 6348, pp. 56-58, 1991.

[16] T. M. Willey, C. Bostedt, T. van Buuren et al., "Molecular limits to the quantum confinement model in diamond clusters," Physical Review Letters, vol. 95, no. 11, Article ID 113401, 2005.

[17] D. C. Elias, R. R. Nair, T. M. G. Mohiuddin et al., "Control of graphene's properties by reversible hydrogenation: evidence for graphane," Science, vol. 323, no. 5914, pp. 610-613, 2009.

[18] J. Zhou, Q. Wang, Q. Sun, X. S. Chen, Y. Kawazoe, and P. Jena, "Ferromagnetism in semihydrogenated graphene sheet," Nano Letters, vol. 9, no. 11, pp. 3867-3870, 2009.

[19] L. A. Chernozatonskii, P. B. Sorokin, A. A. Kuzubov et al., "Influence of size effect on the electronic and elastic properties of diamond films with nanometer thickness," Journal of Physical Chemistry C, vol. 115, no. 1, pp. 132-136, 2011.

[20] J. Li, H. D. Li, Z. G. Wang, and G. T. Zou, "Structure, magnetic, and electronic properties of hydrogenated two-dimensional diamond films," Applied Physics Letters, vol. 102, no. 7, Article ID 073114, 2013.

[21] M. Fyta, "Nitrogen-vacancy centers and dopants in ultrathin diamond films: electronic structure," Journal of Physical Chemistry C, vol. 117, no. 41, pp. 21376-21381, 2013.

[22] D. Odkhuu, D. Shin, R. S. Ruoff, and N. Park, "Conversion of multilayer graphene into continuous ultrathin $\mathrm{sp}^{3}$-bonded carbon films on metal surfaces," Scientific Reports, vol. 3, article 3276, 2013

[23] A. G. Kvashnin, L. A. Chernozatonskii, B. I. Yakobson, and P. B. Sorokin, "Phase diagram of quasi-two-dimensional carbon, from graphene to diamond," Nano Letters, vol. 14, no. 2, pp. 676681,2014

[24] L. Y. Antipina and P. B. Sorokin, "Converting chemically functionalized few-layer graphene to diamond films: a computational study," The Journal of Physical Chemistry C, vol. 119, no. 5, pp. 2828-2836, 2015.

[25] K. Honda, T. N. Rao, D. A. Tryk et al., "Impedance characteristics of the nanoporous honeycomb diamond electrodes for electrical double-layer capacitor applications," Journal of the Electrochemical Society, vol. 148, no. 7, pp. A668-A679, 2001.

[26] Y. S. Zou, Y. Yang, W. J. Zhang et al., "Fabrication of diamond nanopillars and their arrays," Applied Physics Letters, vol. 92, no. 5, Article ID 053105, 2008.

[27] J. Song, H. D. Li, F. Lin, L. Y. Wang, H. L. Wu, and Y. Q. Yang, "Plasmon-enhanced photoluminescence of Si-V centers in diamond from a nanoassembled metal-diamond hybrid structure," CrystEngComm, vol. 16, no. 36, pp. 8356-8362, 2014.

[28] J. Song, S. H. Cheng, H. D. Li, H. Y. Guo, S. P. Xu, and W. Q. Xu, "A novel surface-enhanced Raman scattering substrate: diamond nanopit infilled with gold nanoparticle," Materials Letters, vol. 135, pp. 214-217, 2014.

[29] J. Song, H. D. Li, S. H. Cheng, and Q. L. Wang, "Fabrication of a hybrid structure of diamond nanopits infilled with a gold nanoparticle," RSC Advances, vol. 4, no. 60, pp. 32000-32003, 2014.

[30] N. J. Yang, H. Uetsuka, E. Osawa, and C. E. Nebel, "Vertically aligned nanowires from boron-doped diamond," Nano Letters, vol. 8, no. 11, pp. 3573-3576, 2008.

[31] N. J. Yang, H. Uetsuka, and C. E. Nebel, "Biofunctionalization of vertically aligned diamond nanowires," Advanced Functional Materials, vol. 19, no. 6, pp. 887-893, 2009.

[32] C. Lu, Y. L. Li, S. B. Tian, W. X. Li, J. J. Li, and C. Z. Gu, "Enhanced gas-sensing by diamond nanoneedle arrays formed by reactive ion etching," Microelectronic Engineering, vol. 88, no. 8, pp. 2319-2321, 2011.

[33] B. J. M. Hausmann, M. Khan, Y. Zhang et al., "Fabrication of diamond nanowires for quantum information processing applications," Diamond \& Related Materials, vol. 19, no. 5-6, pp. 621-629, 2010.

[34] T. M. Babinec, B. J. M. Hausmann, M. Khan et al., "A diamond nanowire single-photon source," Nature Nanotechnology, vol. 5, no. 3, pp. 195-199, 2010.

[35] Q. Q. Jiang, D. Q. Liu, G. Q. Liu et al., "Focused-ion-beam overlay-patterning of three-dimensional diamond structures for advanced single-photon properties," Journal of Applied Physics, vol. 116, no. 4, Article ID 044308, 2014. 
[36] E. Neu, P. Appel, M. Ganzhorn et al., "Photonic nano-structures on (111)-oriented diamond," Applied Physics Letters, vol. 104, no. 15, Article ID 153108, 2014.

[37] M. Y. Liao, S. Hishita, E. Watanabe, S. Koizumi, and Y. Koide, "Suspended single-crystal diamond nanowires for high-performance nanoelectromechanical switches," Advanced Materials, vol. 22, no. 47, pp. 5393-5397, 2010.

[38] H. D. Li, H. Lü, D. D. Sang et al., "Synthesizing of $\mathrm{ZnO}$ micro/ nanostructures at low temperature with new reducing agents," Chinese Physics Letters, vol. 25, no. 10, pp. 3794-3797, 2008.

[39] H. D. Li, D. D. Sang, S. H. Cheng et al., "Epitaxial growth of $\mathrm{ZnO}$ nanorods on diamond and negative differential resistance of n-ZnO nanorod/p-diamond heterojunction," Applied Surface Science, vol. 280, pp. 201-206, 2013.

[40] D. D. Sang, H. D. Li, and S. H. Cheng, "Growth and electron field emission of $\mathrm{ZnO}$ nanorods on diamond films," Applied Surface Science, vol. 258, no. 1, pp. 333-336, 2011.

[41] Q. Yu, J. Li, H. D. Li, Q. L. Wang, S. H. Cheng, and L. L. Li, "Fabrication, structure, and photocatalytic activities of borondoped $\mathrm{ZnO}$ nanorods hydrothermally grown on CVD diamond film," Chemical Physics Letters, vol. 539-540, pp. 74-78, 2012.

[42] A. Hachigo, H. Nakahata, K. Higaki, S. Fujii, and S.-I. Shikata, "Heteroepitaxial growth of $\mathrm{ZnO}$ films on diamond (111) plane by magnetron sputtering," Applied Physics Letters, vol. 65, no. 20, pp. 2556-2558, 1994.

[43] H. B. Yu, S. Chen, X. Quan, H. M. Zhao, and Y. B. Zhang, "Fabrication of $\mathrm{a} \mathrm{TiO}_{2}-\mathrm{BDD}$ heterojunction and its application as a photocatalyst for the simultaneous oxidation of an azo dye and reduction of $\mathrm{Cr}(\mathrm{VI})$, , Environmental Science and Technology, vol. 42, no. 10, pp. 3791-3796, 2008.

[44] J. H. Qu and X. Zhao, "Design of $\mathrm{BDD}-\mathrm{TiO}_{2}$ hybrid electrode with P-N function for photoelectroatalytic degradation of organic contaminants," Environmental Science and Technology, vol. 42, no. 13, pp. 4934-4939, 2008.

[45] Y. S. Chen, J. C. Crittenden, S. Hackney, L. Sutter, and D. W. Hand, "Preparation of a novel $\mathrm{TiO}_{2}$-based p-n junction nanotube photocatalyst," Environmental Science \& Technology, vol. 39, no. 5, pp. 1201-1208, 2005.

[46] H. G. Kim, P. H. Borse, W. Choi, and J. S. Lee, "Photocatalytic nanodiodes for visible-light photocatalysis," Angewandte Chemie-International Edition, vol. 44, no. 29, pp. 4585-4589, 2005.

[47] F. Marken, A. S. Bhambra, D.-H. Kim, R. J. Mortimer, and S. J. Stott, "Electrochemical reactivity of $\mathrm{TiO}_{2}$ nanoparticles adsorbed onto boron-doped diamond surfaces," Electrochemistry Communications, vol. 6, no. 11, pp. 1153-1158, 2004.

[48] D. V. Bavykin, E. V. Milsom, F. Marken et al., "A novel cationbinding $\mathrm{TiO}_{2}$ nanotube substrate for electro- and bioelectrocatalysis," Electrochemistry Communications, vol. 7, no. 10, pp. 1050-1058, 2005.

[49] K. Siuzdak, R. Bogdanowicz, M. Sawczak, and M. Sobaszek, "Enhanced capacitance of composite $\mathrm{TiO}_{2}$ nanotube/borondoped diamond electrodes studied by impedance spectroscopy," Nanoscale, vol. 7, no. 2, pp. 551-558, 2015.

[50] F. Schuster, M. Hetzl, S. Weiszer et al., "Position-controlled growth of $\mathrm{GaN}$ nanowires and nanotubes on diamond by molecular beam epitaxy," Nano Letters, vol. 15, no. 3, pp. 17731779, 2015.

[51] F. Schuster, A. Winnerl, S. Weiszer, M. Hetzl, J. A. Garrido, and M. Stutzmann, "Doped GaN nanowires on diamond: structural properties and charge carrier distribution," Journal of Applied Physics, vol. 117, no. 4, Article ID 044307, 2015.
[52] Ü. Özgür, Y. I. Alivov, C. Liu et al., "A comprehensive review of ZnO materials and devices," Journal of Applied Physics, vol. 98, no. 4, Article ID 041301, 2005.

[53] C. X. Wang, G. W. Yang, T. C. Zhang et al., "Fabrication of transparent $\mathrm{p}-\mathrm{n}$ hetero-junction diodes by $\mathrm{p}$-diamond film and n-ZnO film," Diamond and Related Materials, vol. 12, no. 9, pp. 1548-1552, 2003.

[54] A. B. Djuriić, A. M. C. Ng, and X. Y. Chen, "ZnO nanostructures for optoelectronics: material properties and device applications," Progress in Quantum Electronics, vol. 34, no. 4, pp. 191-259, 2010.

[55] N. K. Reddy, Q. Ahsanulhaq, J. H. Kim, and Y. B. Hahn, "Behavior of $n-\mathrm{ZnO}$ nanorods/ $p$-Si heterojunction devices at higher temperatures," Applied Physics Letters, vol. 92, no. 4, Article ID 043127, 2008.

[56] X.-M. Zhang, M.-Y. Lu, Y. Zhang, L.-J. Chen, and Z. L. Wang, "Fabrication of a high-brightness blue-light-emitting diode using a $\mathrm{ZnO}$-Nanowire array grown on $\mathrm{p}-\mathrm{GaN}$ thin film," Advanced Materials, vol. 21, no. 27, pp. 2767-2770, 2009.

[57] J. B. Baxter and E. S. Aydil, "Epitaxial growth of $\mathrm{ZnO}$ nanowires on a- and c-plane sapphire," Journal of Crystal Growth, vol. 274, no. 3-4, pp. 407-411, 2005.

[58] T. Ochiai and A. Fujishima, "Photoelectrochemical properties of $\mathrm{TiO}_{2}$ photocatalyst and its applications for environmental purification," Journal of Photochemistry and Photobiology C: Photochemistry Reviews, vol. 13, no. 4, pp. 247-262, 2012. 

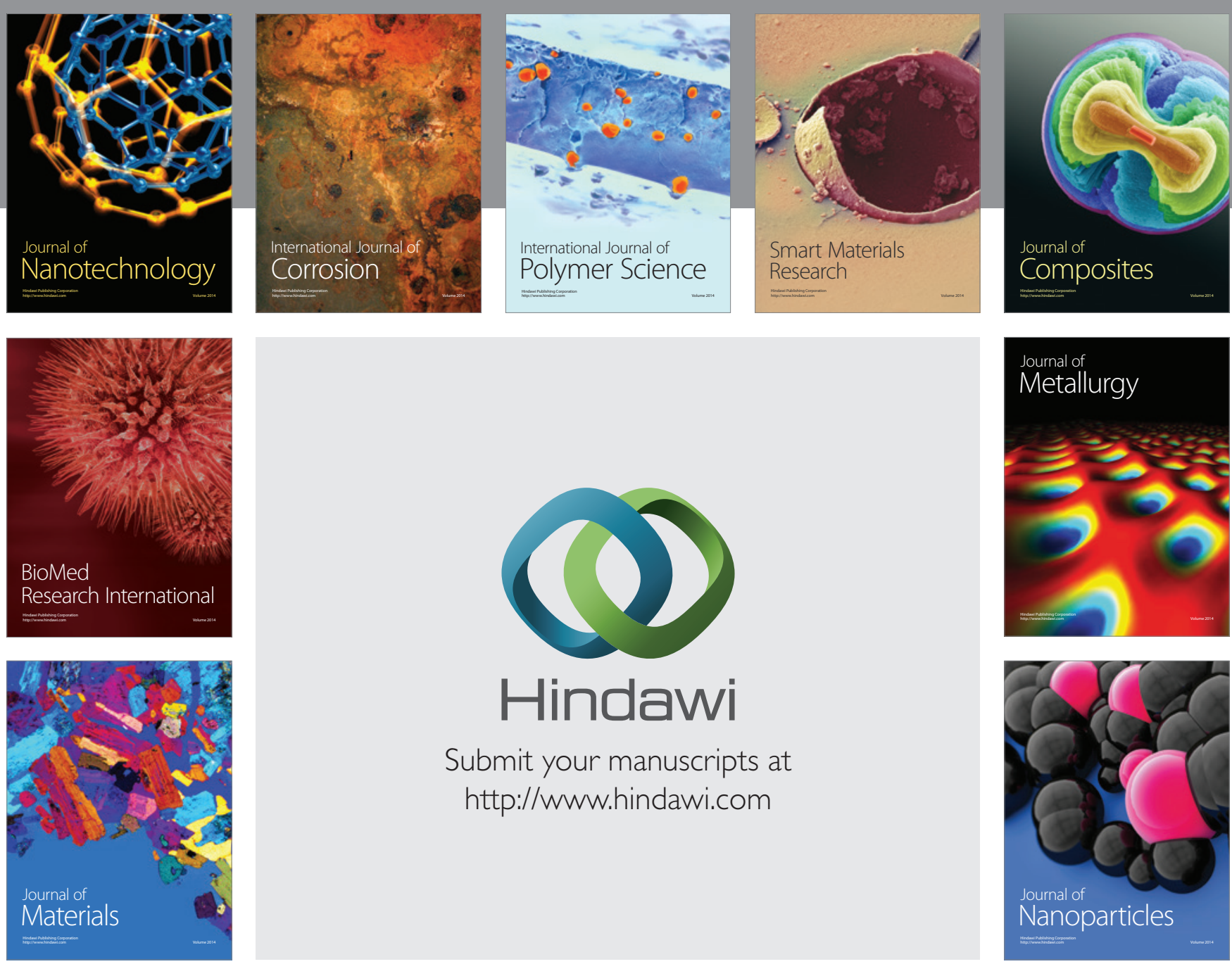

Submit your manuscripts at http://www.hindawi.com
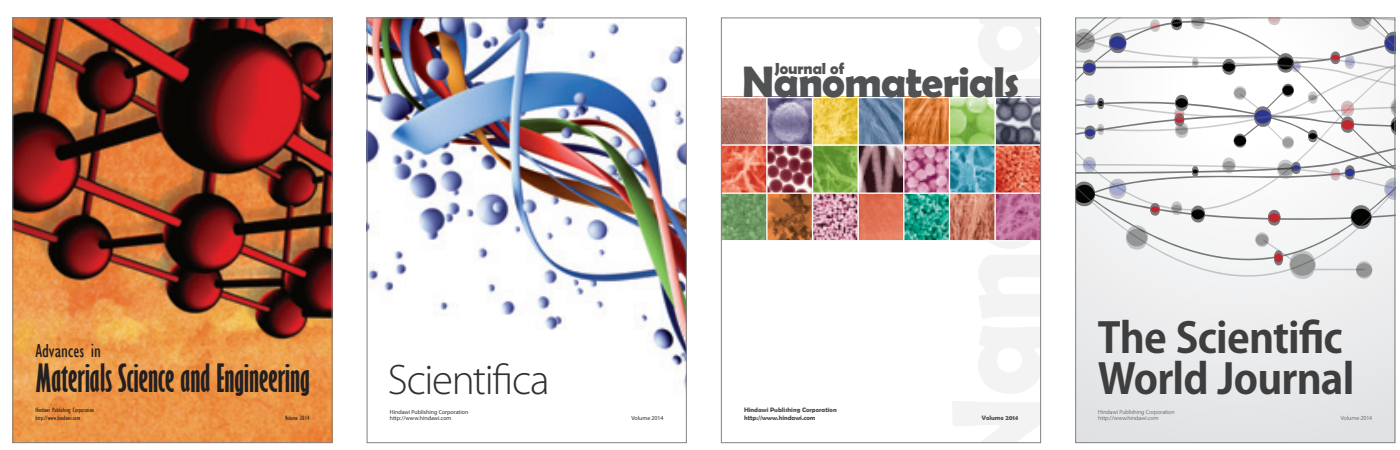

\section{The Scientific World Journal}
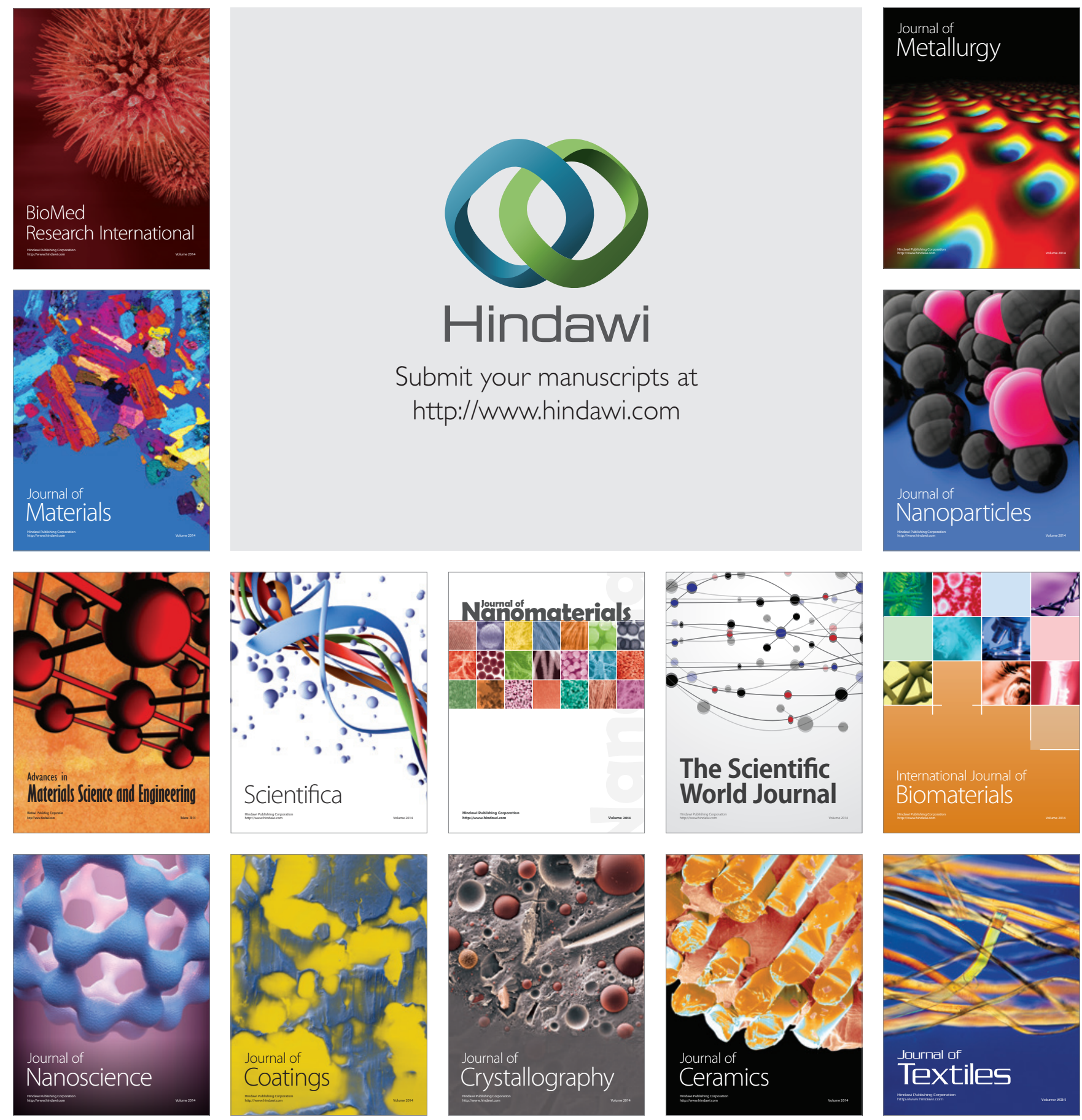